Supporting Information

\title{
Structure-Activity Relationships of Anti-microRNA Oligonucleotides Containing Cationic Guanidine-Modified Nucleic Acids
}

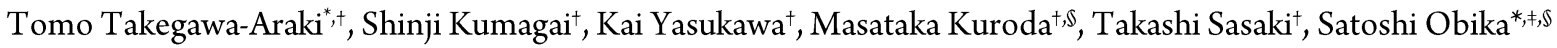

+Sohyaku. Innovative Research Division, Mitsubishi Tanabe Pharma Corporation, Muraoka-Higashi, Fujisawa, Kanagawa, 251-8555, Japan

${ }^{\ddagger}$ Graduate School of Pharmaceutical Sciences, Osaka University, 1-6 Yamadaoka, Suita, Osaka, 565-0871, Japan.

${ }^{\S}$ National Institutes of Biomedical Innovation, Health and Nutrition (NIBIOHN), 7-6-8 Saito-Asagi, Ibaraki, Osaka 5670085, Japan.

${ }^{*}$ Corresponding Authors Email:

takegawa.tomo@ma.mt-pharma.co.jp (Tomo Takegawa-Araki); obika@phs.osaka-u.ac.jp (Satoshi Obika)

\section{Table of Contents}

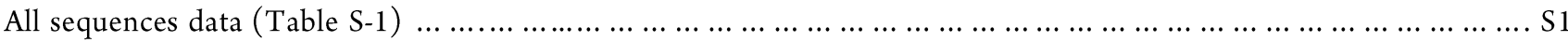

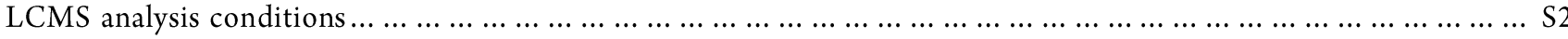

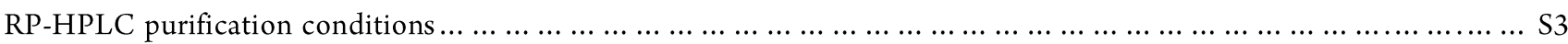

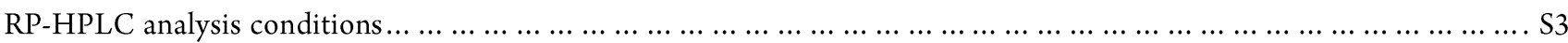

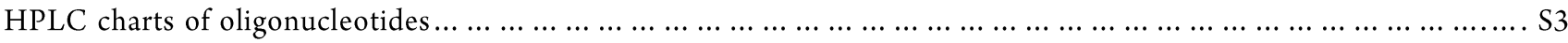

Representative UV melting curves (Figure S-1, Table S-2) $\ldots \ldots \ldots \ldots \ldots \ldots \ldots \ldots \ldots \ldots \ldots \ldots \ldots \ldots \ldots \ldots \ldots \ldots \ldots \ldots \ldots \ldots$

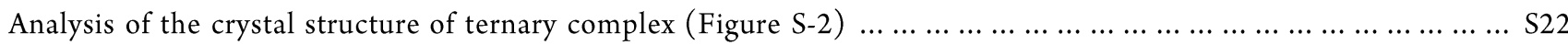

All sequences data

\begin{tabular}{|c|c|c|c|c|c|c|}
\hline Seq No. & Sequence (5' to 3') & Modification & Calcd. mass & Found. mass & $\begin{array}{c}\text { HPLC Purity } \\
\text { (UV \%) }\end{array}$ & HPLC Retention Time (min) \\
\hline AMO-1 & AaCatCagTctGatAagCT & LNA & 6350.13 & 6352.34 & 95.86 & 10.755 \\
\hline AMO-2 & AaCatCagTctGatAagCT & GuNA & 6678.57 & 6679.16 & 100.00 & $15.376,15.669$ \\
\hline AMO-3 & AaCatCagTctGaTaaGcT & GuNA & 6664.54 & 6662.72 & 100.00 & 15.203 \\
\hline AMO-4 & AaCatCagTctGAtaAgcT & GuNA & 6664.54 & 6666.49 & 98.13 & 15.107 \\
\hline AMO-5 & AAcaTcaGtcTgatAagCT & GuNA & 6650.51 & 6649.87 & 99.16 & $15.280,15.645$ \\
\hline AMO-6 & AAcaTcaGtcTgaTaaGcT & GuNA & 6636.49 & 6635.17 & 100.00 & $15.352,15.695$ \\
\hline AMO-7 & AAcaTcaGtcTgAtaAgcT & GuNA & 6636.49 & 6635.69 & 100.00 & 15.133 \\
\hline AMO-8 & AacAtcAgtCtgatAagCT & GuNA & 6595.48 & 6594.54 & 100.00 & 15.797 \\
\hline AMO-9 & AacAtcAgtCtgaTaaGcT & GuNA & 6581.45 & 6580.73 & 100.00 & 15.744 \\
\hline AMO-10 & AacAtcAgtCtgAtaAgcT & GuNA & 6581.45 & 6582.46 & 100.00 & 15.771 \\
\hline
\end{tabular}




\begin{tabular}{|c|c|c|c|c|c|c|}
\hline AMO-11 & AAcaTcaGtcTgataaGCT & GuNA & 6636.49 & 6649.95 & 100.00 & $15.432,15.778,16.102$ \\
\hline AMO-12 & AAcaTcaGtcTgataAgCT & GuNA & 6650.51 & 6651.49 & 100.00 & $15.457,15.802,16.094$ \\
\hline AMO-13 & AAcaTcaGtcTgaTaagCT & GuNA & 6650.51 & 6650.64 & 94.98 & 15.231 \\
\hline AMO-14 & AAcaTcaGtcTgAtaagCT & GuNA & 6650.51 & 6650.01 & 100.00 & $15.242,15.585$ \\
\hline AMO-15 & AAcaTcaGtcTgataAGcT & GuNA & 6636.49 & 6637.01 & 100.00 & $15.342,15.705$ \\
\hline AMO-16 & AAcaTcaGtcTgatAaGcT & GuNA & 6636.49 & 6635.80 & 98.18 & $15.229,15.630$ \\
\hline AMO-17 & AAcaTcaGtcTgAtaaGcT & GuNA & 6636.49 & 6633.89 & 94.15 & $15.176,15.534$ \\
\hline AMO-18 & AAcaTcaGtcTgatAAgcT & GuNA & 6636.49 & 6635.24 & 96.06 & 15.221 \\
\hline AMO-19 & AAcaTcaGtcTgaTaAgcT & GuNA & 6636.49 & 6635.80 & 98.78 & 15.131 \\
\hline AMO-20 & AAcaTcaGtcTgaTAagcT & GuNA & 6636.49 & 6637.53 & 100.00 & 15.136 \\
\hline AMO-21 & AAcaTcaGtcTgAtAagcT & GuNA & 6636.49 & 6635.62 & 98.50 & 15.182 \\
\hline AMO-22 & AAcaTcaGtcTgaTaaGctA & GuNA & 6965.76 & 6967.95 & 95.14 & 12.847 \\
\hline AMO-23 & AAcaTcaGtcTgaTaaGctA & GuNA/MOE & 6970.77 & 6969.35 & 100.00 & $16.164,16.370$ \\
\hline AMO-24 & AAcaTcaGtcTgaTaaGcTA & GuNA/MOE & 7039.84 & 7038.80 & 100.00 & 15.697 \\
\hline AMO-25 & AAcaTcagtCtGaTaaGctA & GuNA & 6979.79 & 6982.20 & 93.28 & $12.948,13.256$ \\
\hline AMO-26 & AAcaTcagTctGaTaaGctA & GuNA & 6965.76 & 6968.00 & 97.46 & 12.919 \\
\hline AMO-27 & AAcaTcagtCTgaTaaGctA & GuNA & 6979.79 & 6981.69 & 94.86 & 12.977 \\
\hline AMO-28 & AAcaTcagTcTgaTaaGctA & GuNA & 6965.76 & 6968.83 & 96.96 & 12.831 \\
\hline AMO-29 & AAcaTcagTCtgaTaaGctA & GuNA & 6979.79 & 6981.59 & 98.07 & 12.989 \\
\hline AMO-30 & AAcaTcaGtCtgaTaaGctA & GuNA & 6979.79 & 6981.85 & 97.43 & 12.964 \\
\hline AMO-31 & AAcaTcaGTctgaTaaGctA & GuNA & 6965.76 & 6968.34 & 98.89 & $12.995,13.321$ \\
\hline AMO-32 & AaCaTcagTcTgaTaaGctA & GuNA & 6979.79 & 6982.08 & 98.01 & $12.789,13.133$ \\
\hline AMO-33 & AaCatCagTcTgaTaaGctA & GuNA & 6993.81 & 6996.29 & 98.78 & $12.755,13.103$ \\
\hline
\end{tabular}

Table S-1. Lower case letters in the sequences refer to DNA, and uppercase letters refer to LNA (blue), GuNA (Black) or 2'-

MOE (red) (C denotes methyl cytosine). All phosphodiester linkages were replaced by phosphorothioate (PS) linkages. The measured values were obtained under the conditions shown in S2 to S5. ESI produces multi- ply-charged ions, and found molecular weight were obtained by the transformation of those peaks. Several GuNA sequences had multiple peaks detected by HPLC analysis, in which case the peaks that had same MS were combined to calculate purity.

\section{LCMS analysis conditions}

\section{AMO2 AMO-33}

Waters ZQ mass detector (ESI)

Mobile phase A: $400 \mathrm{mM}$ HFIP / 15mM TEA in water

Mobile phase B: Methanol

Flow rate: $0.2 \mathrm{~mL} / \mathrm{min}$

Gradient condition: $20-30 \%$ in $2.5 \mathrm{~min}$ 
Column: Waters XBridge ${ }^{\mathrm{zs}}$ BEH C18 $1.7 \mu \mathrm{m}(2.1 \times 100 \mathrm{~mm})$

Column oven temperature: $60^{\circ} \mathrm{C}$

Detector: UV $260 \mathrm{~nm}$

\section{RP-HPLC purification conditions}

\section{AMO2 AMO-33}

Mobile phase A: $20 \mathrm{mM}$ Hexylamine acetate in water

Mobile phase B: Acetonitrile

Gradient condition: $10-50 \%$ in $40 \mathrm{~min}$

Flow rate: $4 \mathrm{~mL} / \mathrm{min}$

Column: Waters XBridge ${ }^{\mathrm{ma}}$ Oligonucleotide BEH C18 OBDTM Prep Column, $130 \AA 2.5 \mu \mathrm{m}(10 \times 50 \mathrm{~mm})$

Column oven temperature: $60^{\circ} \mathrm{C}$

Detector: UV $260 \mathrm{~nm}$

\section{RP-HPLC analysis conditions}

\section{AMO-1 (purchased from GeneDesign)}

Mobile phase A: 100 mM HFIP / 8mM TEA in water

Mobile phase B: Methanol

Flow rate: $1 \mathrm{~mL} / \mathrm{min}$

Column: Waters XBridge ${ }^{\text {ts }}$ BEH C18 $2.5 \mu \mathrm{m}(4.6 \times 75 \mathrm{~mm})$

Column oven temperature: $60^{\circ} \mathrm{C}$

Detector: UV $260 \mathrm{~nm}$

\section{HPLC charts of oligonucleotides}

AMO-1 (gradient condition: B\% 5-30\% in $20 \mathrm{~min}$ )

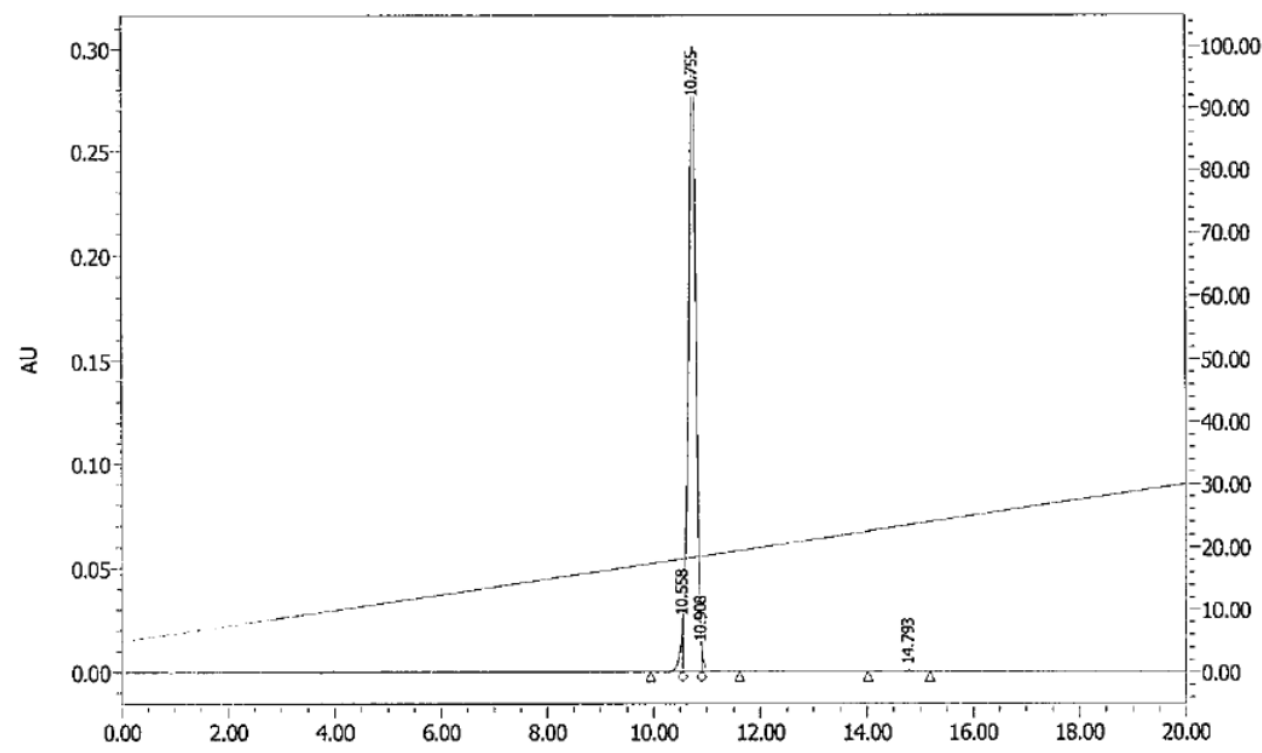

AMO2 AMO-33 
Mobile phase A: $20 \mathrm{mM}$ Hexylamine acetate in water

Mobile phase B: Acetonitrile

Flow rate: $1 \mathrm{~mL} / \mathrm{min}$

Column: Waters XBridge ${ }^{\mathrm{rst}}$ BEH C18 $130 \AA 2.5 \mu \mathrm{m}(4.6 \times 50 \mathrm{~mm})$

Column oven temperature: $60^{\circ} \mathrm{C}$

Detector: UV $260 \mathrm{~nm}$

Blank

(gradient condition: B\% 5-50 \% in $22.5 \mathrm{~min}$, then $100 \%$ in $5 \mathrm{~min}$ )

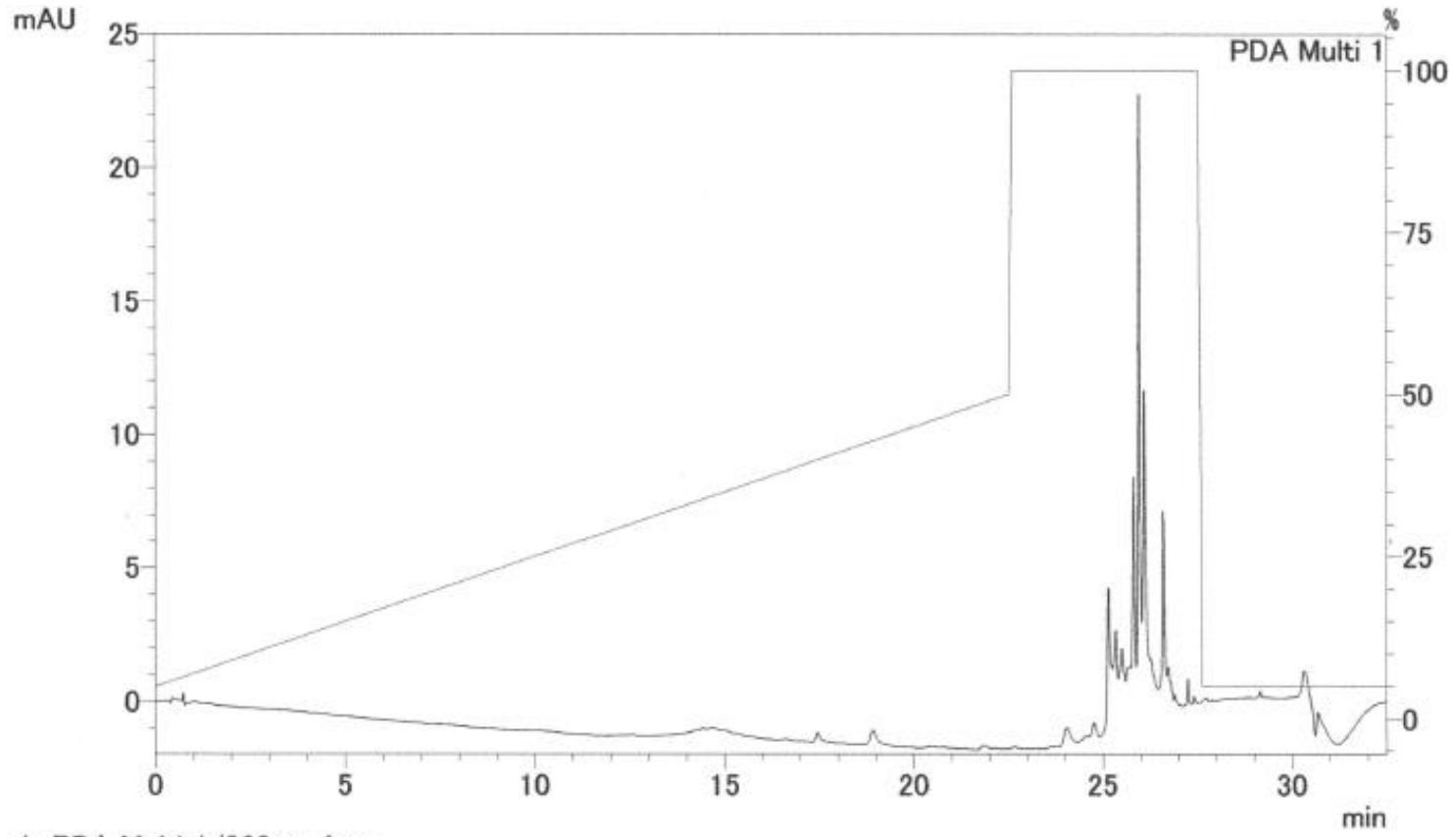

1 PDA Multi $1 / 260 \mathrm{~nm} 4 \mathrm{~nm}$

AMO-2

HPLC (gradient condition: B\% 5-50\% in $22.5 \mathrm{~min}$, then $100 \%$ in $5 \mathrm{~min}$ ) 


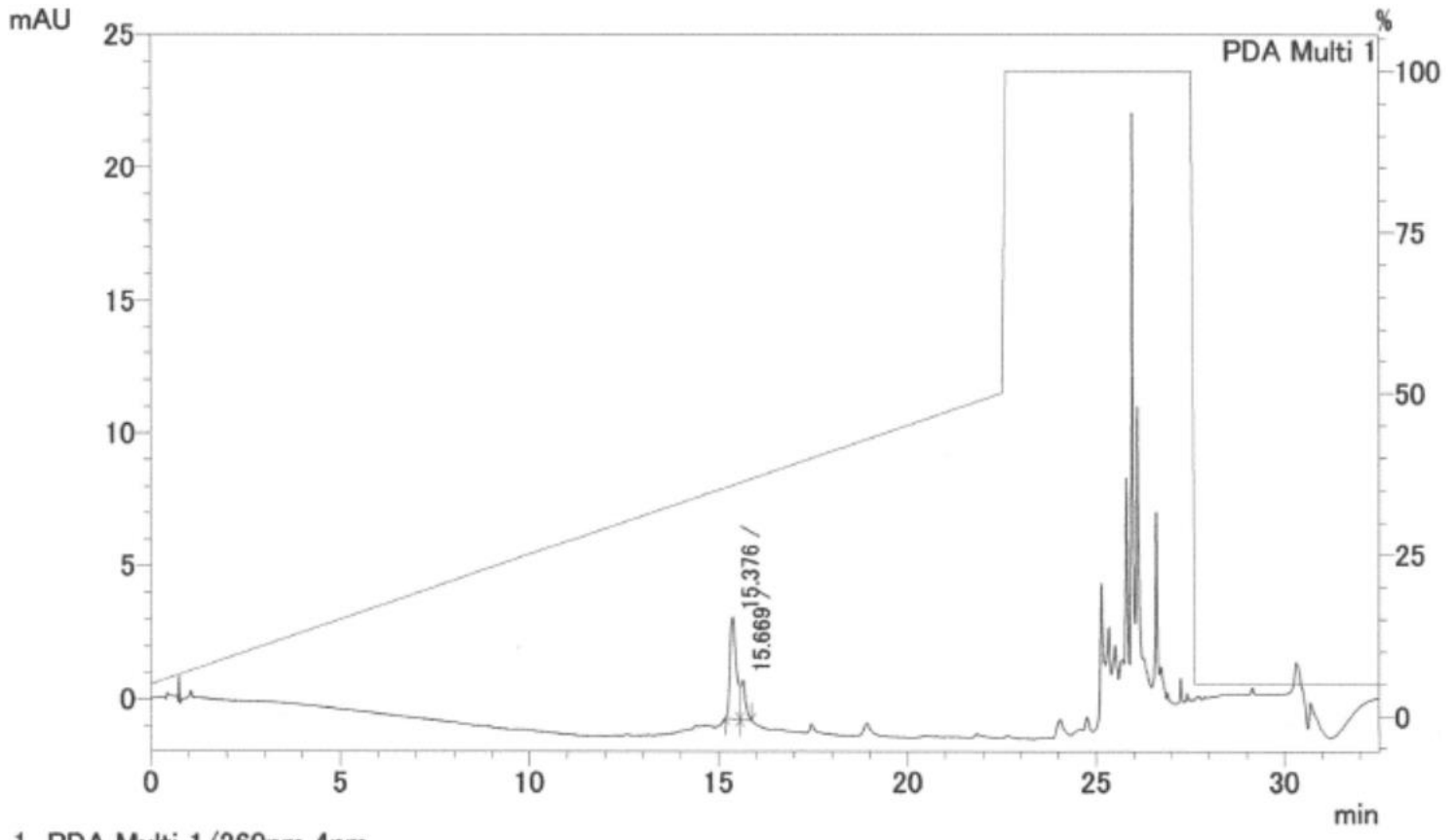

1 PDA Multi $1 / 260 \mathrm{~nm} 4 \mathrm{~nm}$

AMO-3

HPLC (gradient condition: B\% 5-50\% in $22.5 \mathrm{~min}$, then $100 \%$ in $5 \mathrm{~min}$ )

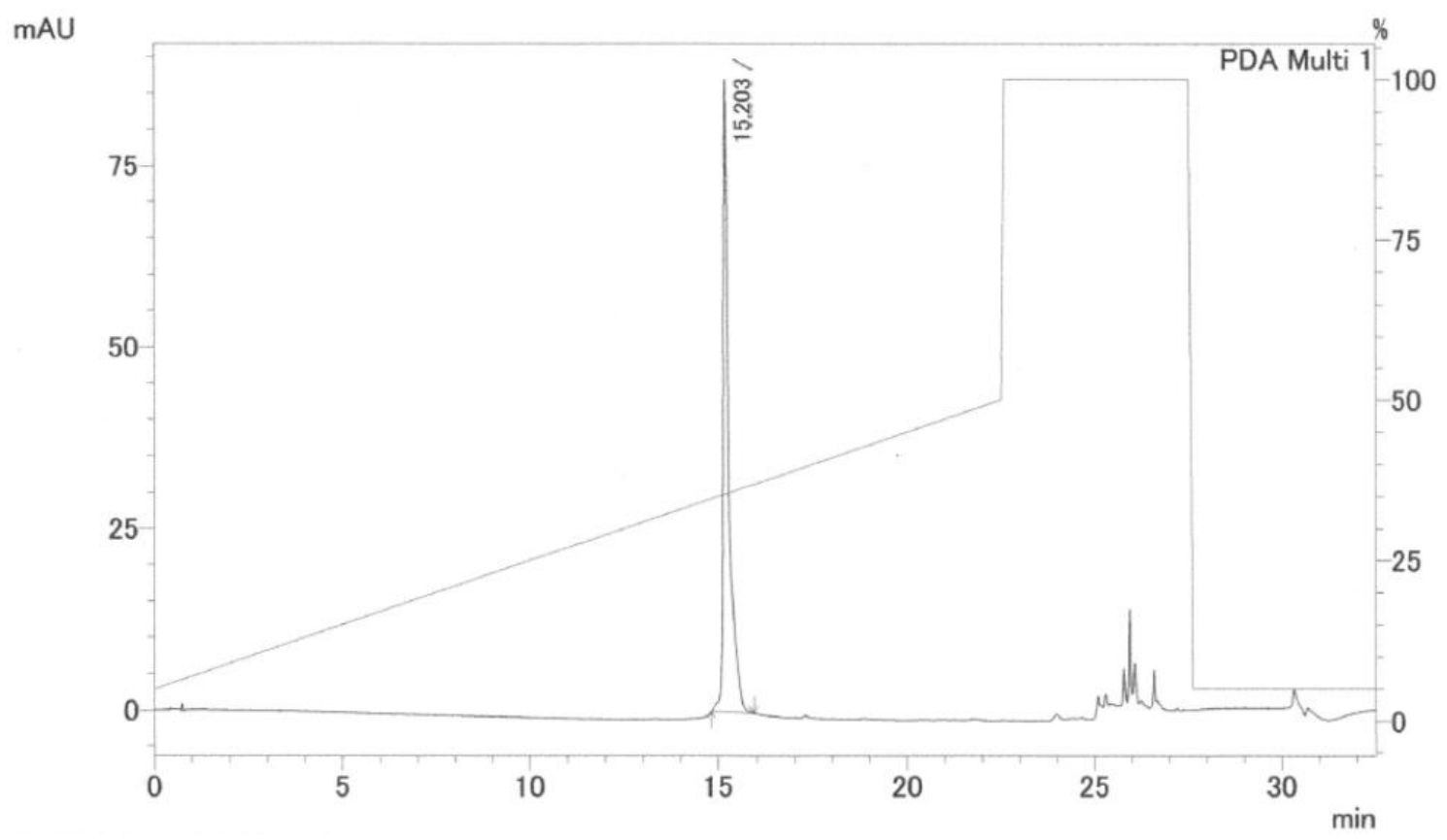

AMO-4

HPLC (gradient condition: B\% 5-50\% in $22.5 \mathrm{~min}$, then $100 \%$ in $5 \mathrm{~min}$ ) 


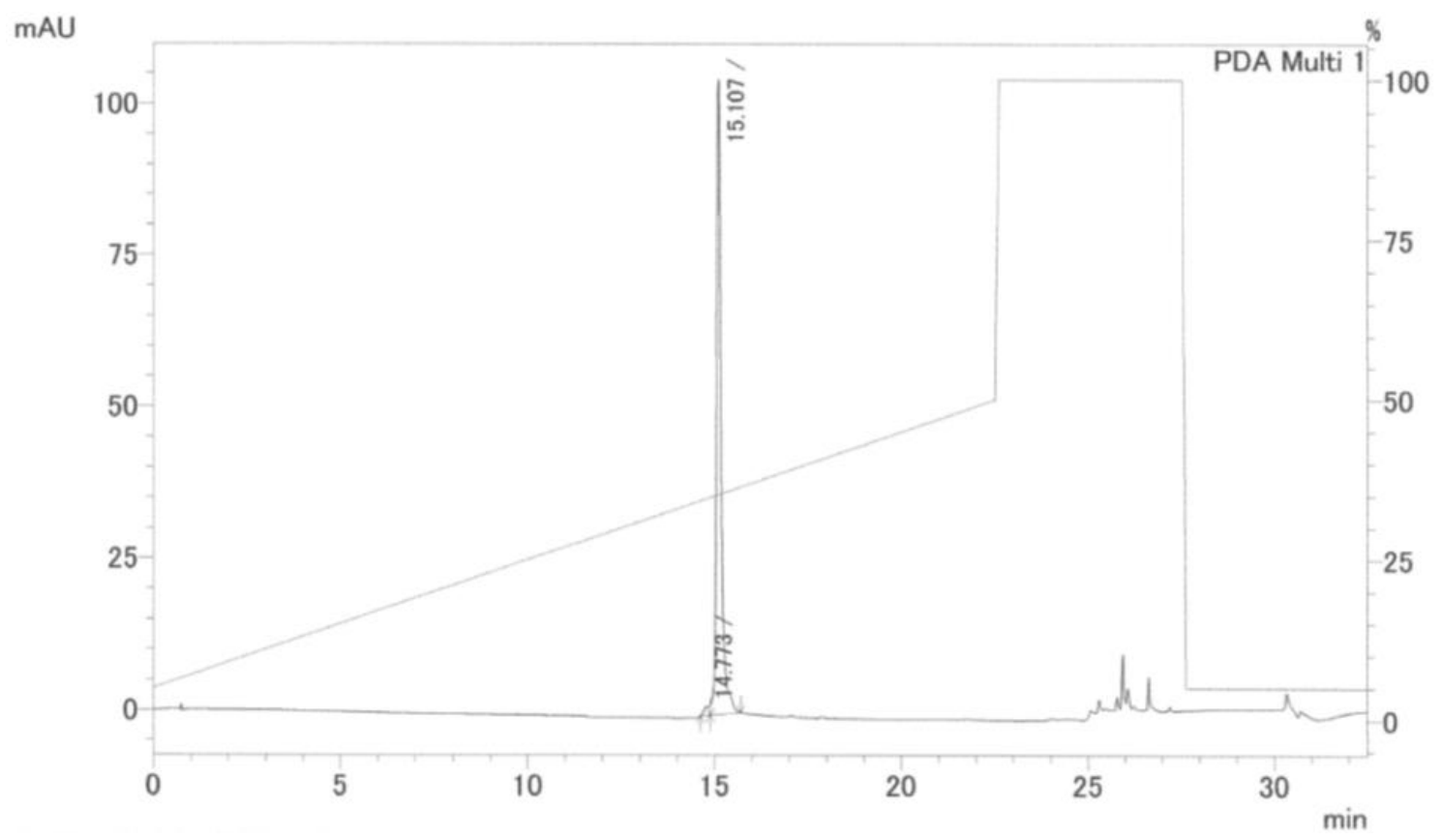

AMO-5

HPLC (gradient condition: B\% 5-50\% in $22.5 \mathrm{~min}$, then $100 \%$ in $5 \mathrm{~min}$ )

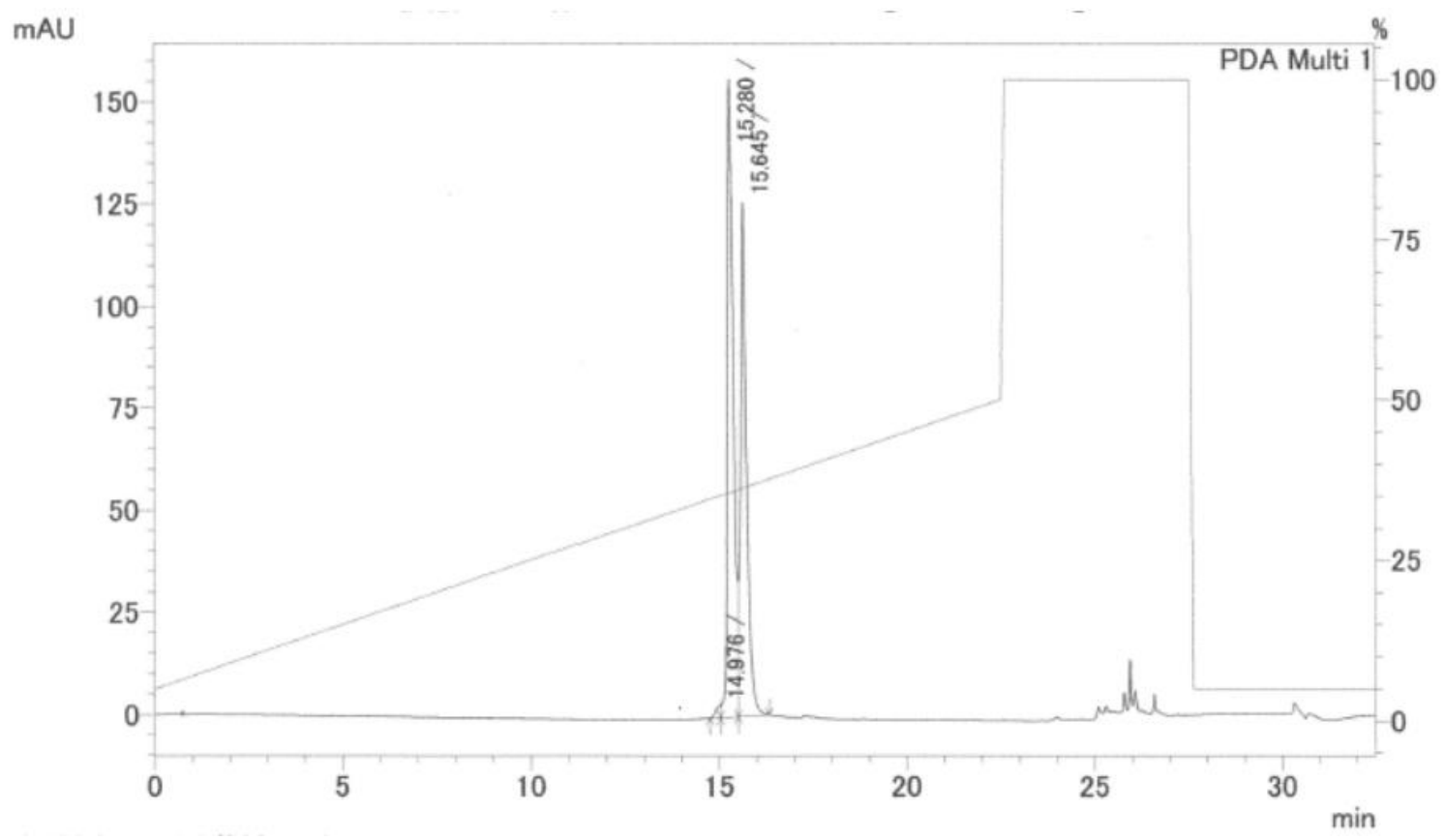

AMO-6

HPLC (gradient condition: B\% 5-50\% in $22.5 \mathrm{~min}$, then $100 \%$ in $5 \mathrm{~min}$ ) 


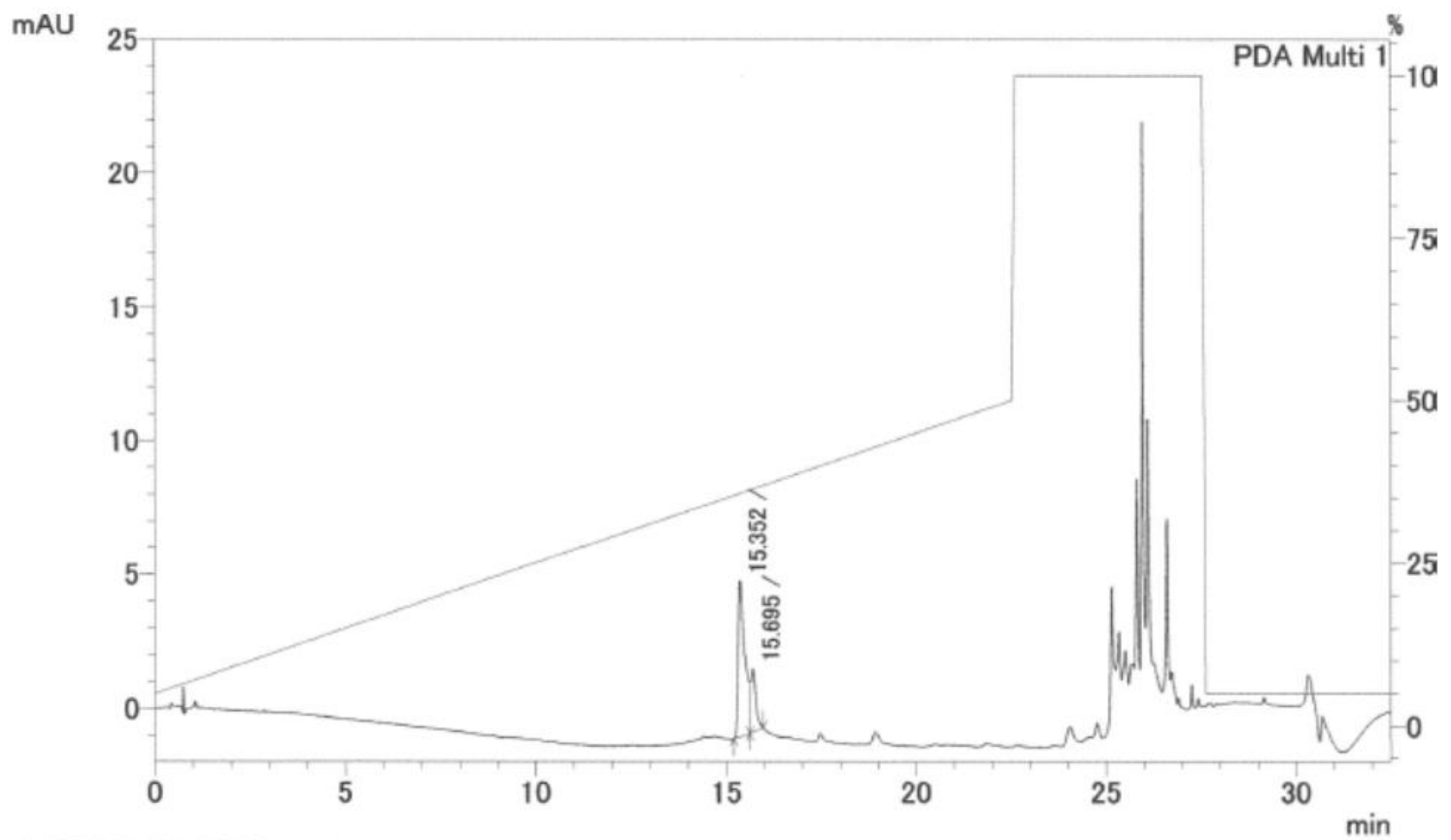

AMO-7

HPLC (gradient condition: B\% 5-50 \% in 22.5 min, then $100 \%$ in 5 min)

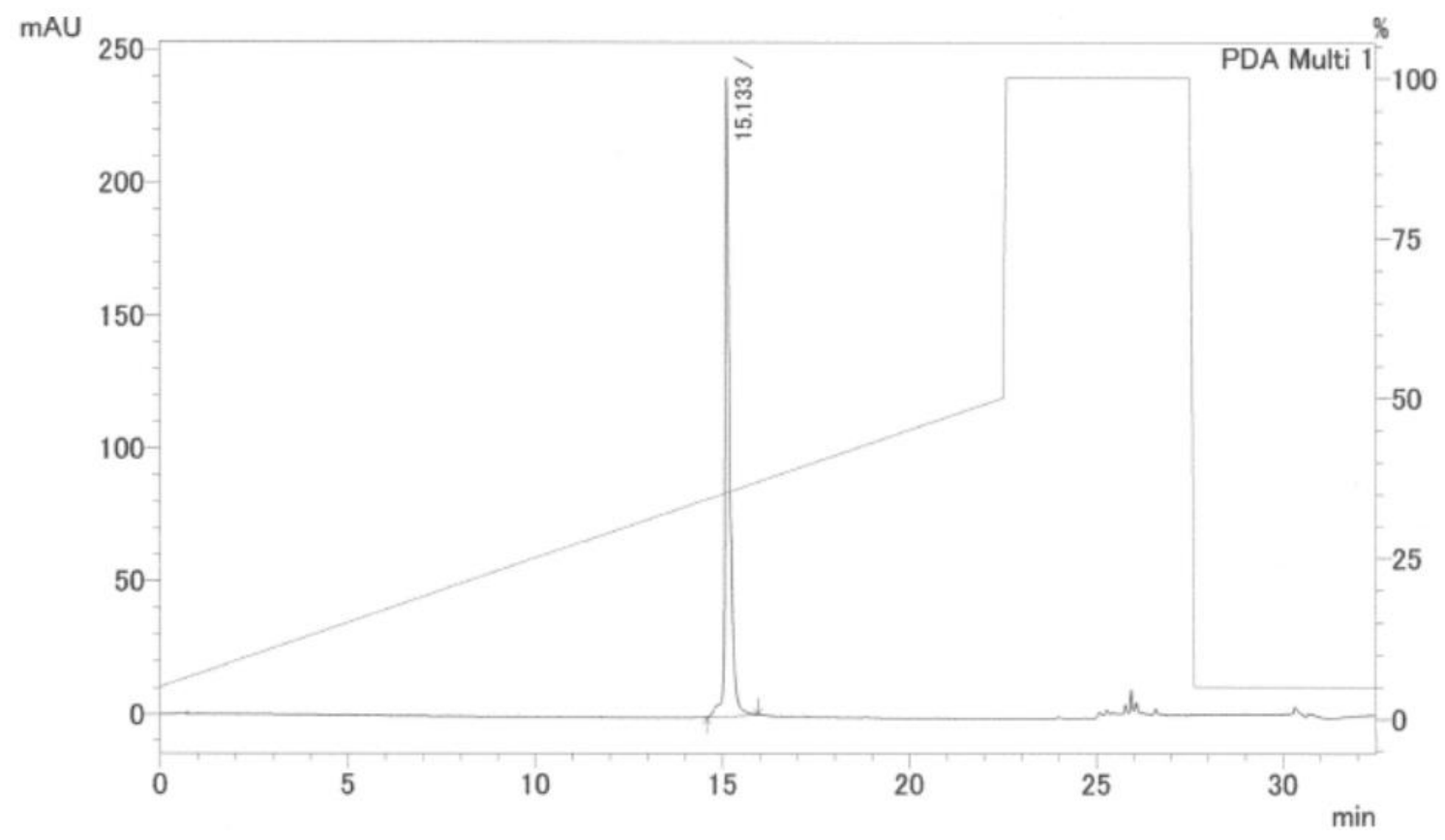

AMO-8

HPLC (gradient condition: B\% 5-50 \% in 22.5 min, then 100\% in 5 min) 


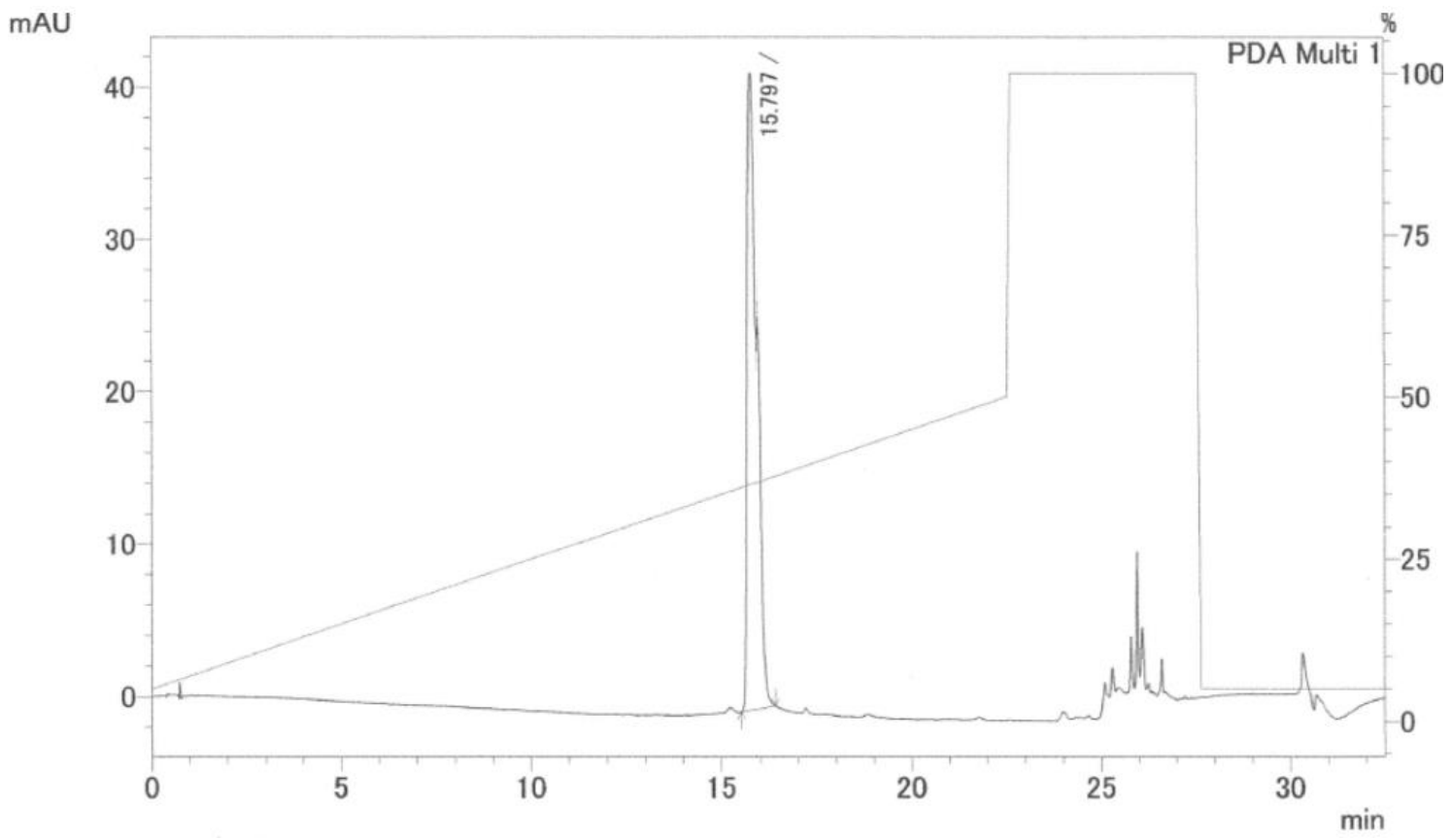

AMO-9

HPLC (gradient condition: B\% 5-50\% in $22.5 \mathrm{~min}$, then $100 \%$ in $5 \mathrm{~min}$ )

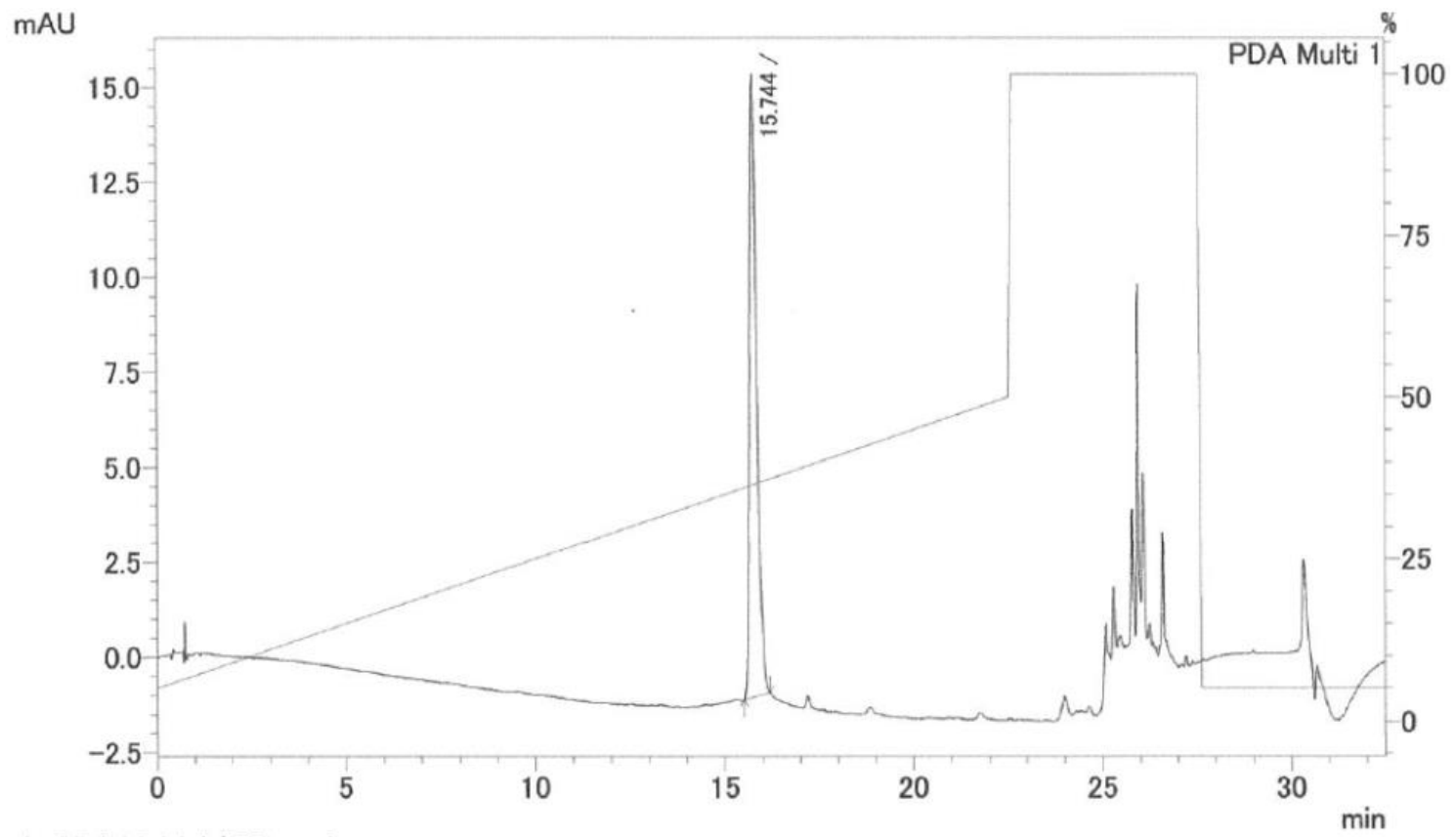

AMO-10

HPLC (gradient condition: B\% 5-50\% in $22.5 \mathrm{~min}$, then $100 \%$ in $5 \mathrm{~min}$ ) 


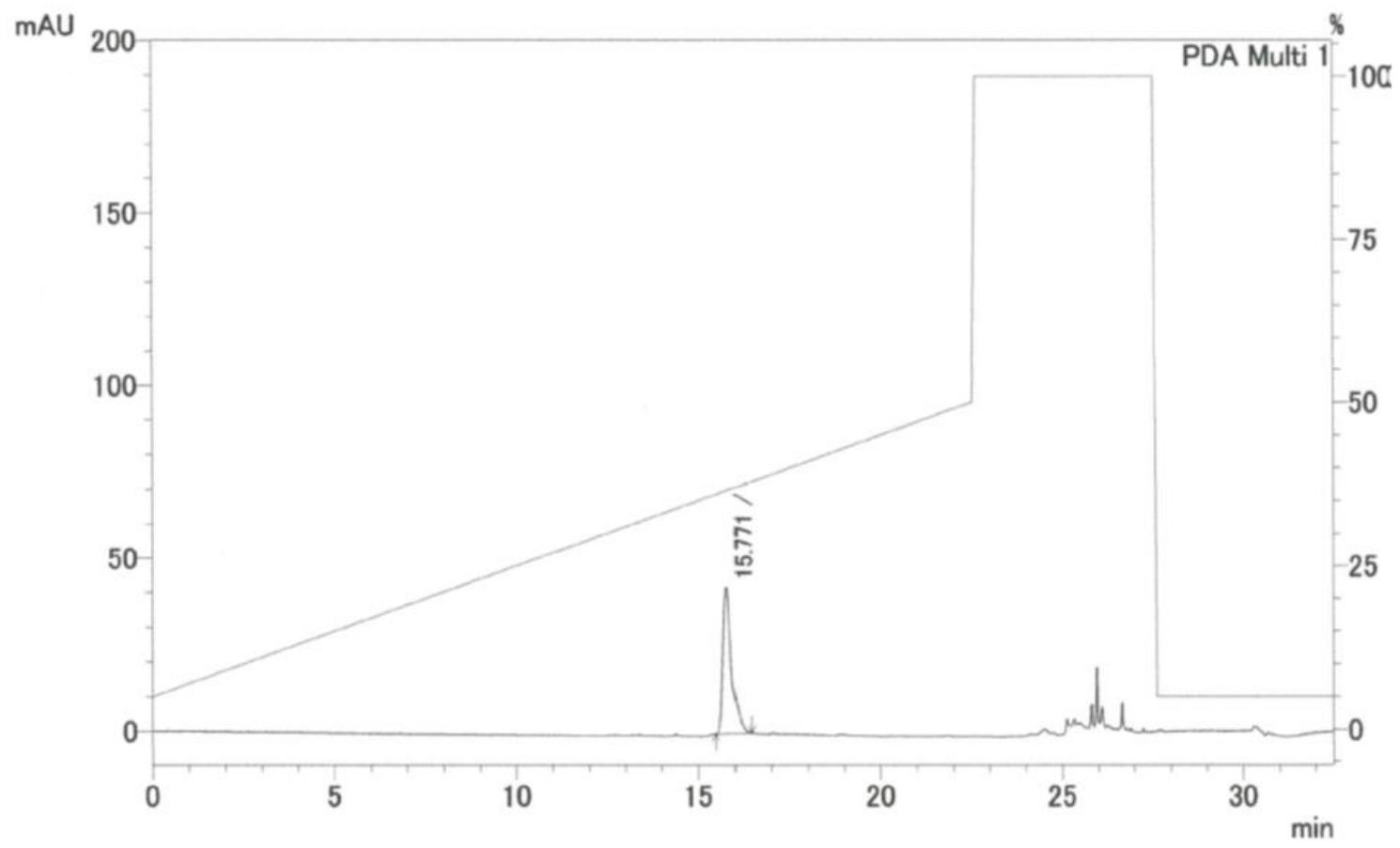

AMO-11

HPLC (gradient condition: B\% 5-50\% in $22.5 \mathrm{~min}$, then $100 \%$ in $5 \mathrm{~min}$ )

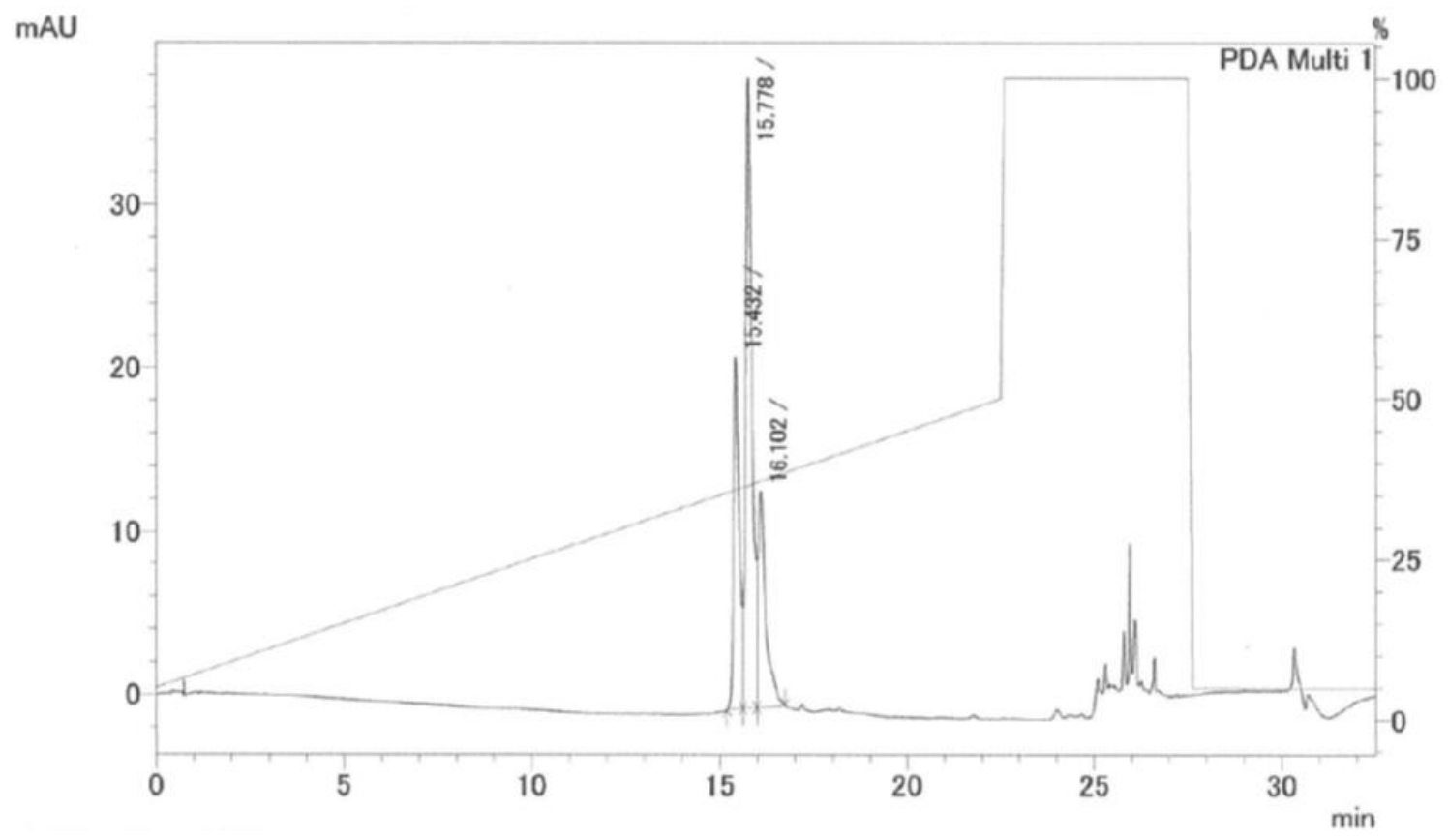

AMO-12

HPLC (gradient condition: B\% 5-50 \% in $22.5 \mathrm{~min}$, then $100 \%$ in $5 \mathrm{~min}$ ) 


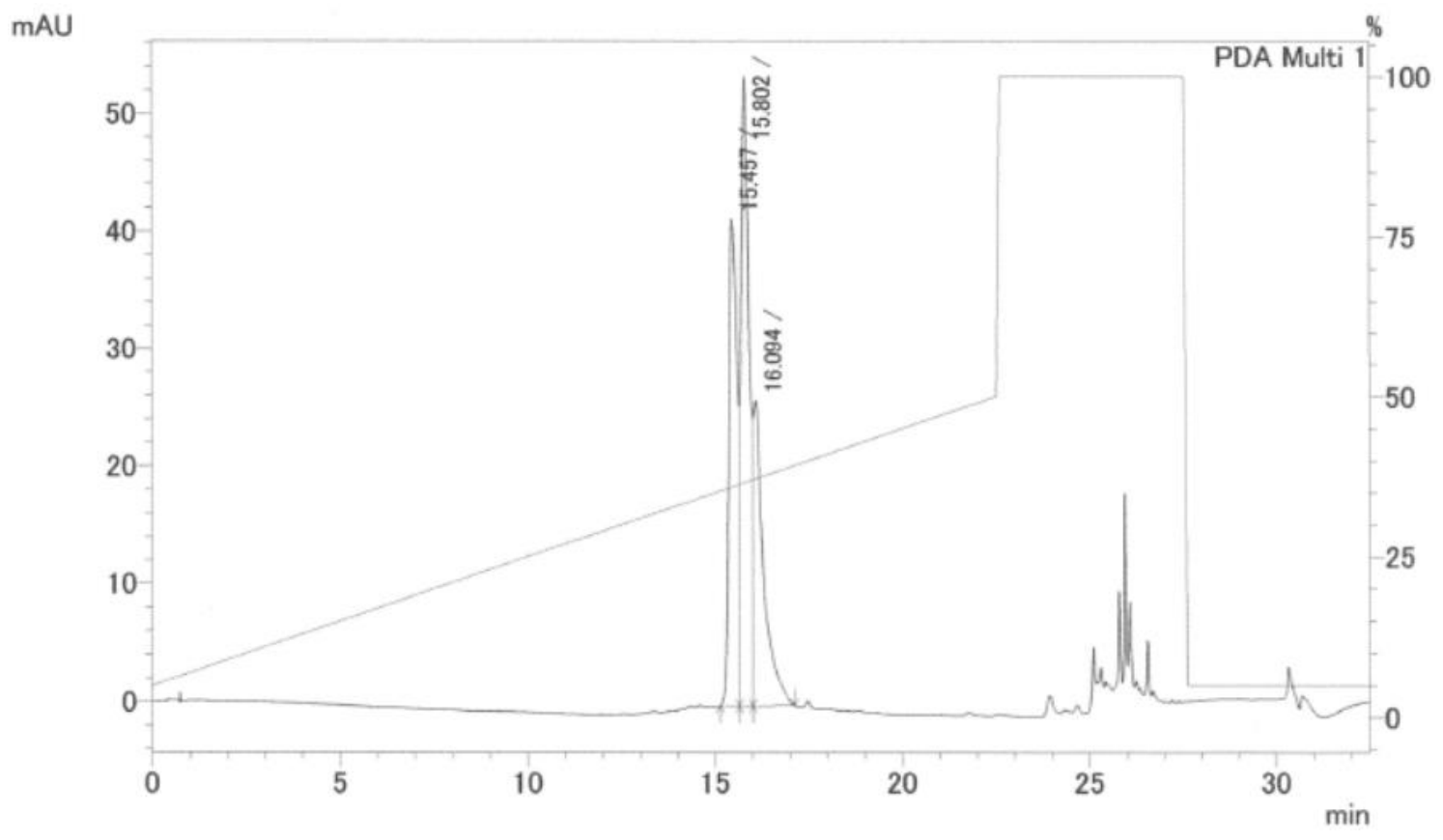

AMO-13

HPLC (gradient condition: B\% 5-50\% in $22.5 \mathrm{~min}$, then $100 \%$ in $5 \mathrm{~min}$ )

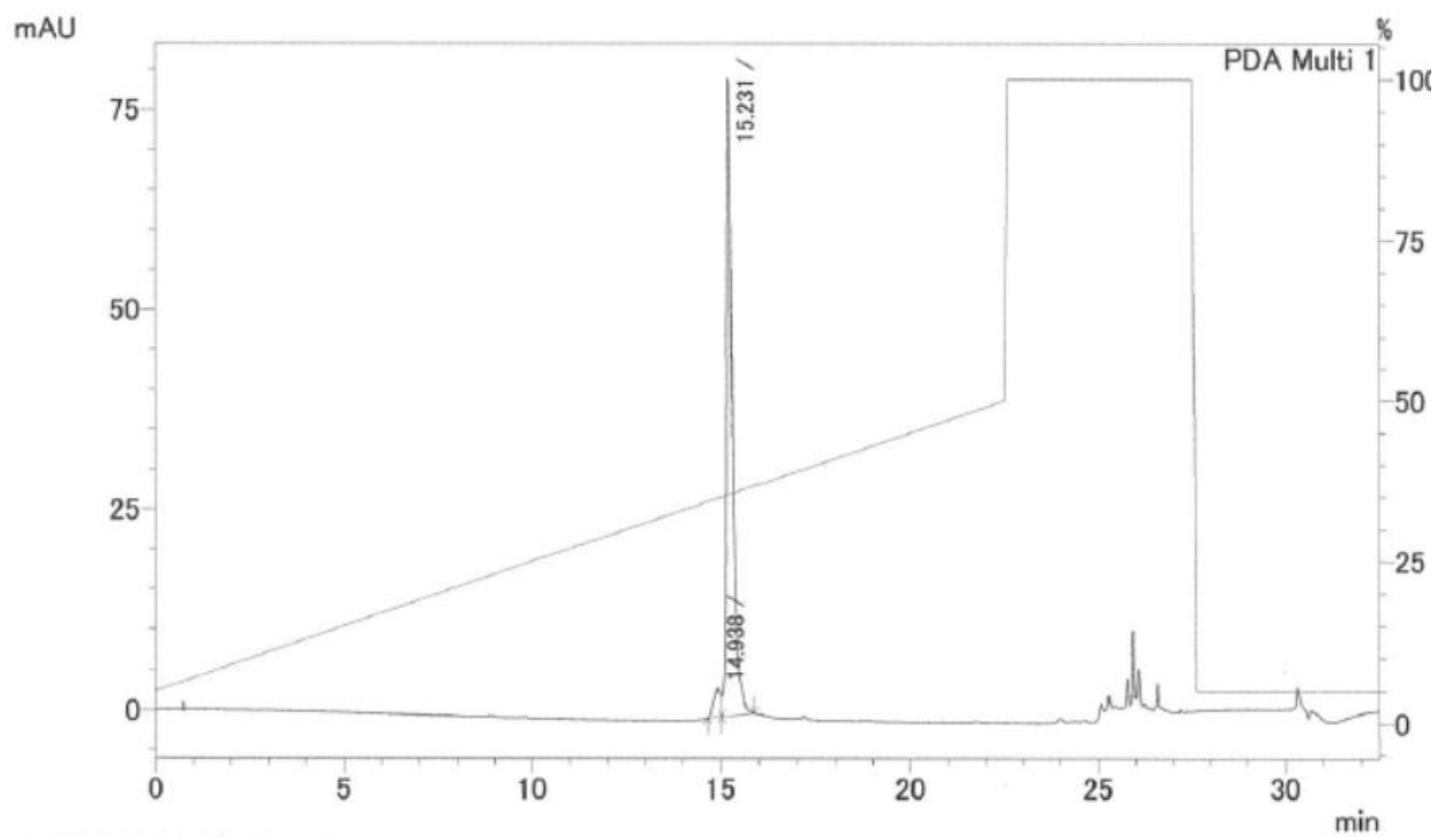

AMO-14

HPLC (gradient condition: B\% 5-50\% in $22.5 \mathrm{~min}$, then $100 \%$ in $5 \mathrm{~min}$ ) 


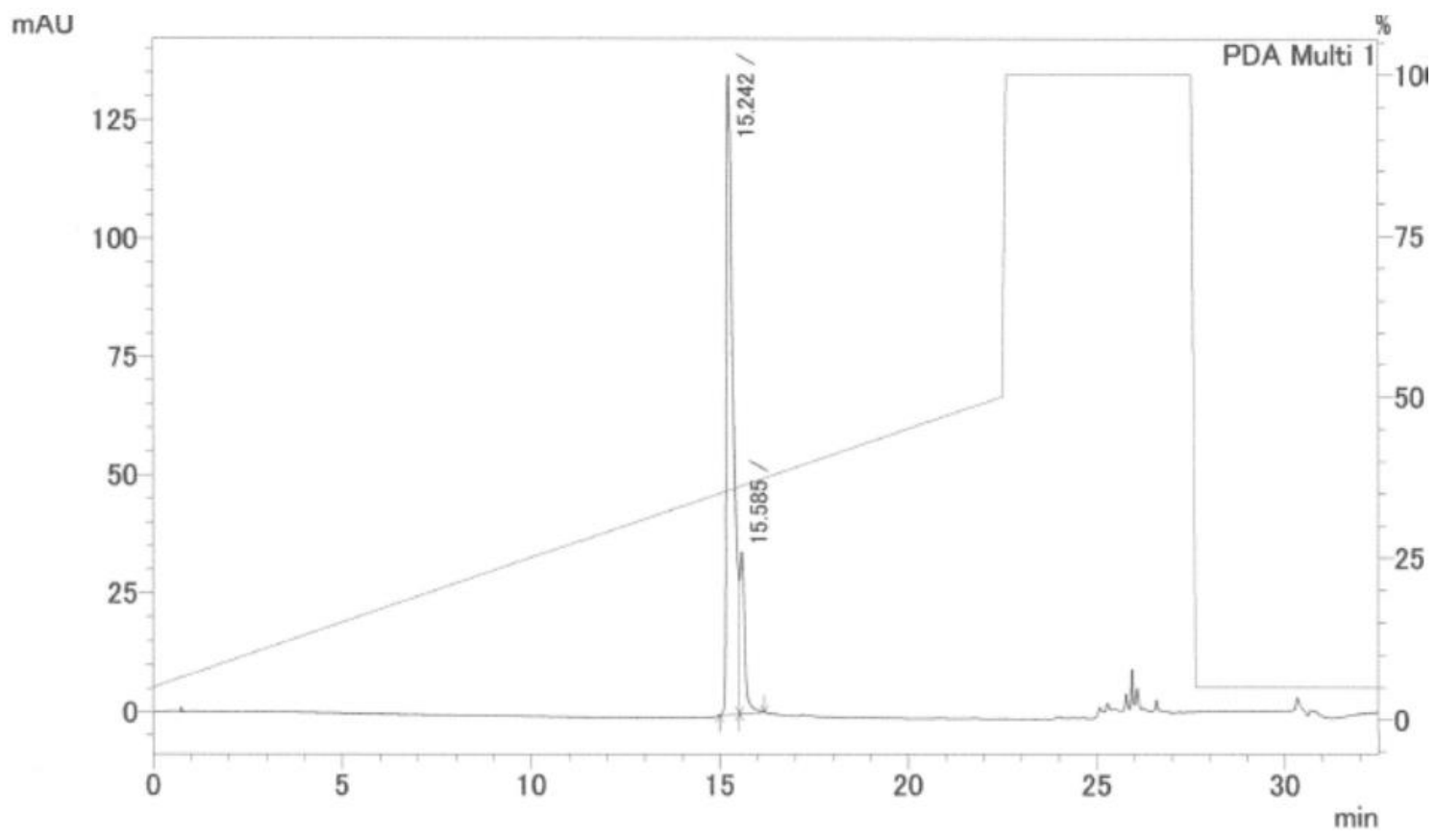

AMO-15

HPLC (gradient condition: B\% 5-50\% in $22.5 \mathrm{~min}$, then $100 \%$ in $5 \mathrm{~min}$ )

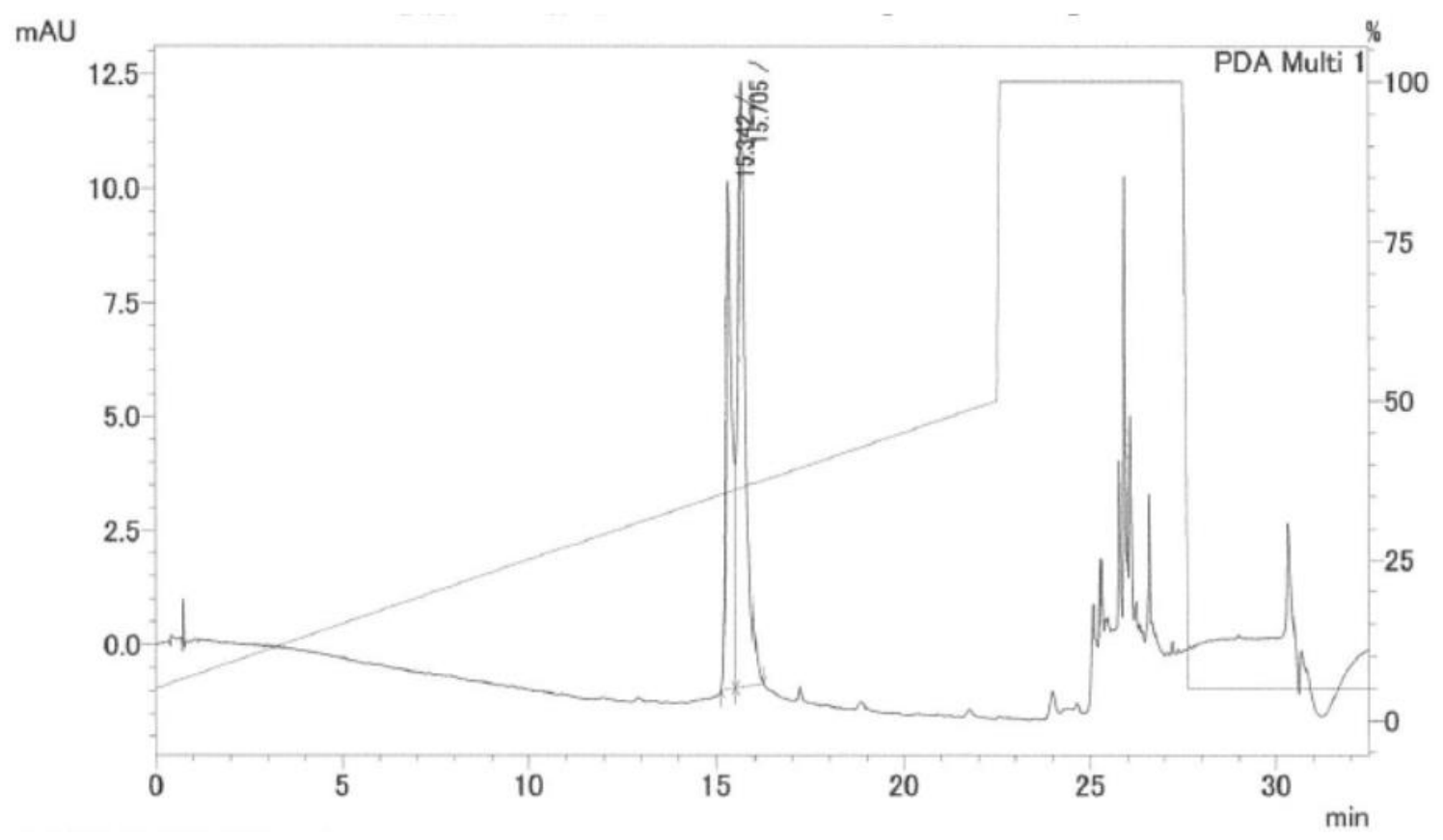

AMO-16

HPLC (gradient condition: B\% 5-50\% in $22.5 \mathrm{~min}$, then $100 \%$ in $5 \mathrm{~min}$ ) 


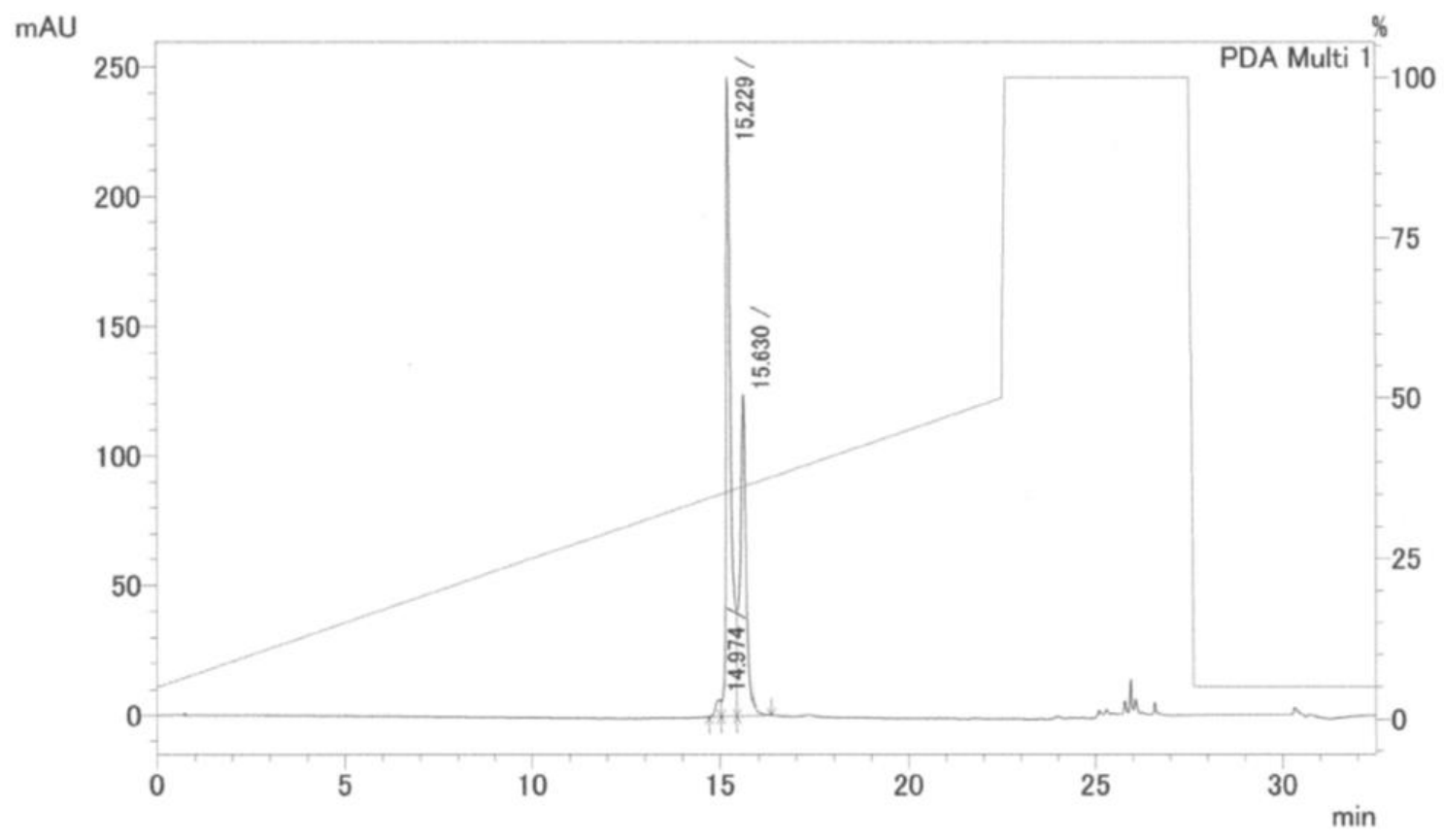

AMO-17

HPLC (gradient condition: B\% 5-50\% in $22.5 \mathrm{~min}$, then $100 \%$ in $5 \mathrm{~min}$ )

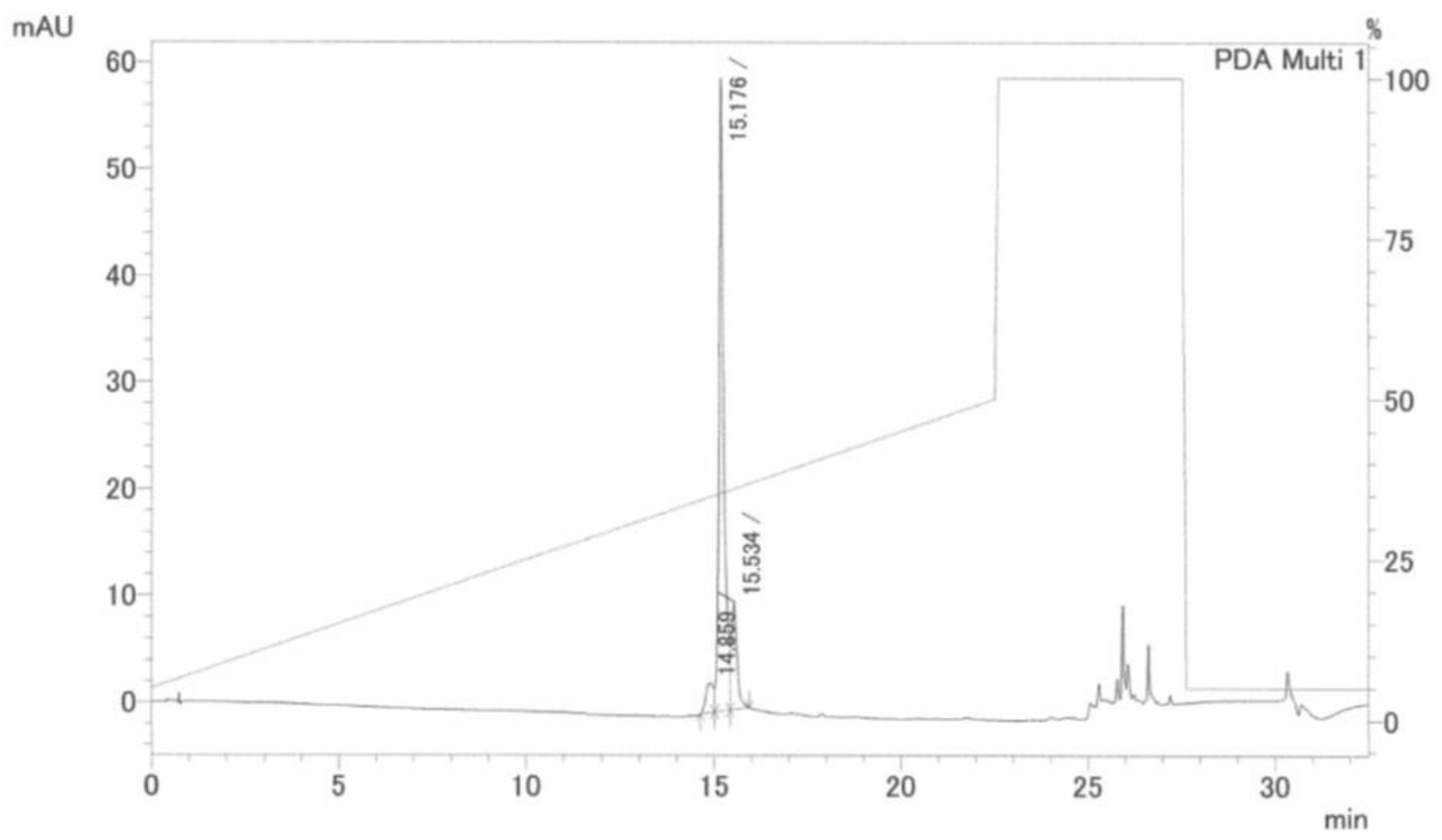

AMO-18

HPLC (gradient condition: B\% 5-50\% in $22.5 \mathrm{~min}$, then $100 \%$ in $5 \mathrm{~min}$ ) 


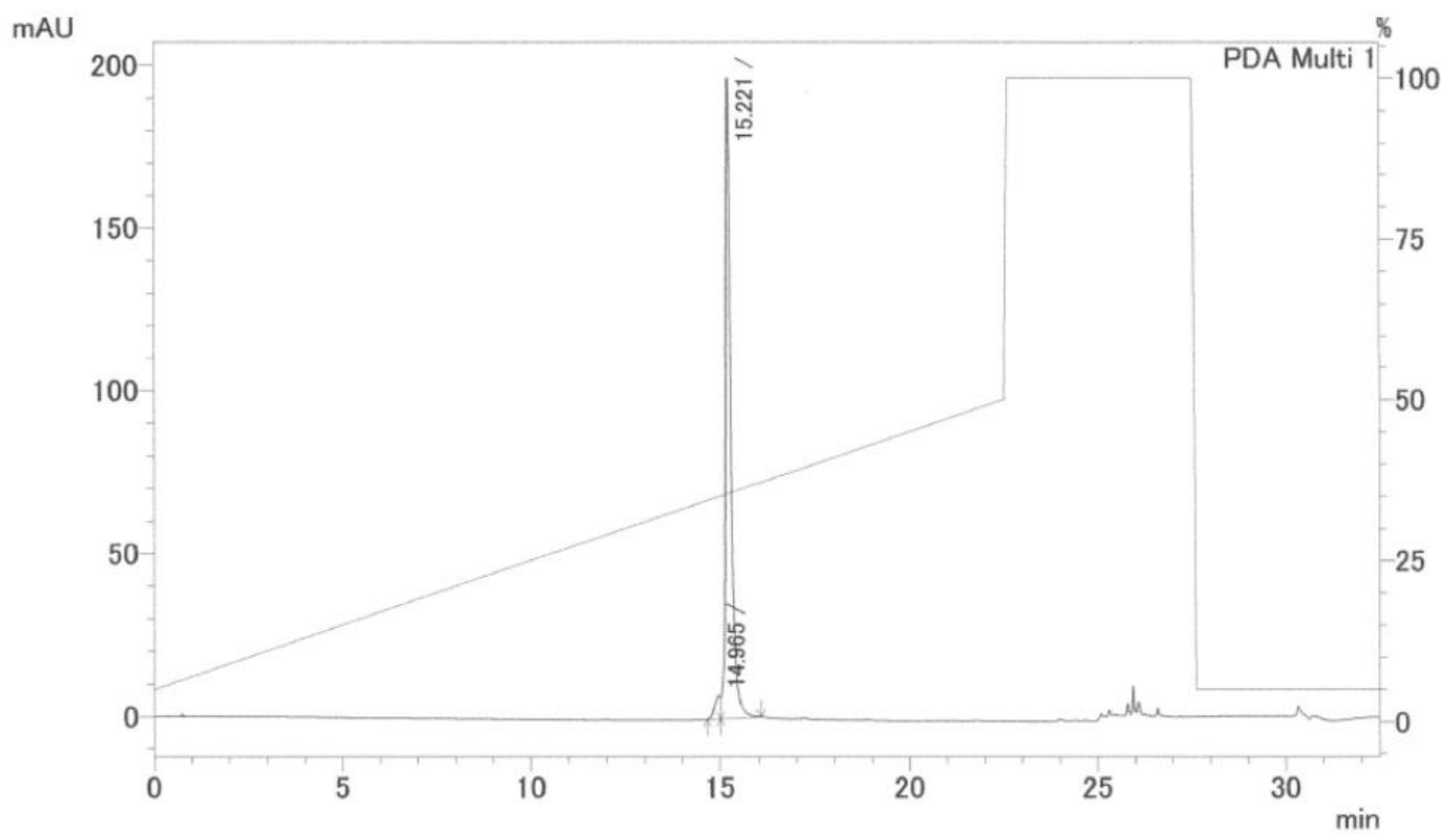

AMO-19

HPLC (gradient condition: B\% 5-50\% in $22.5 \mathrm{~min}$, then $100 \%$ in $5 \mathrm{~min}$ )

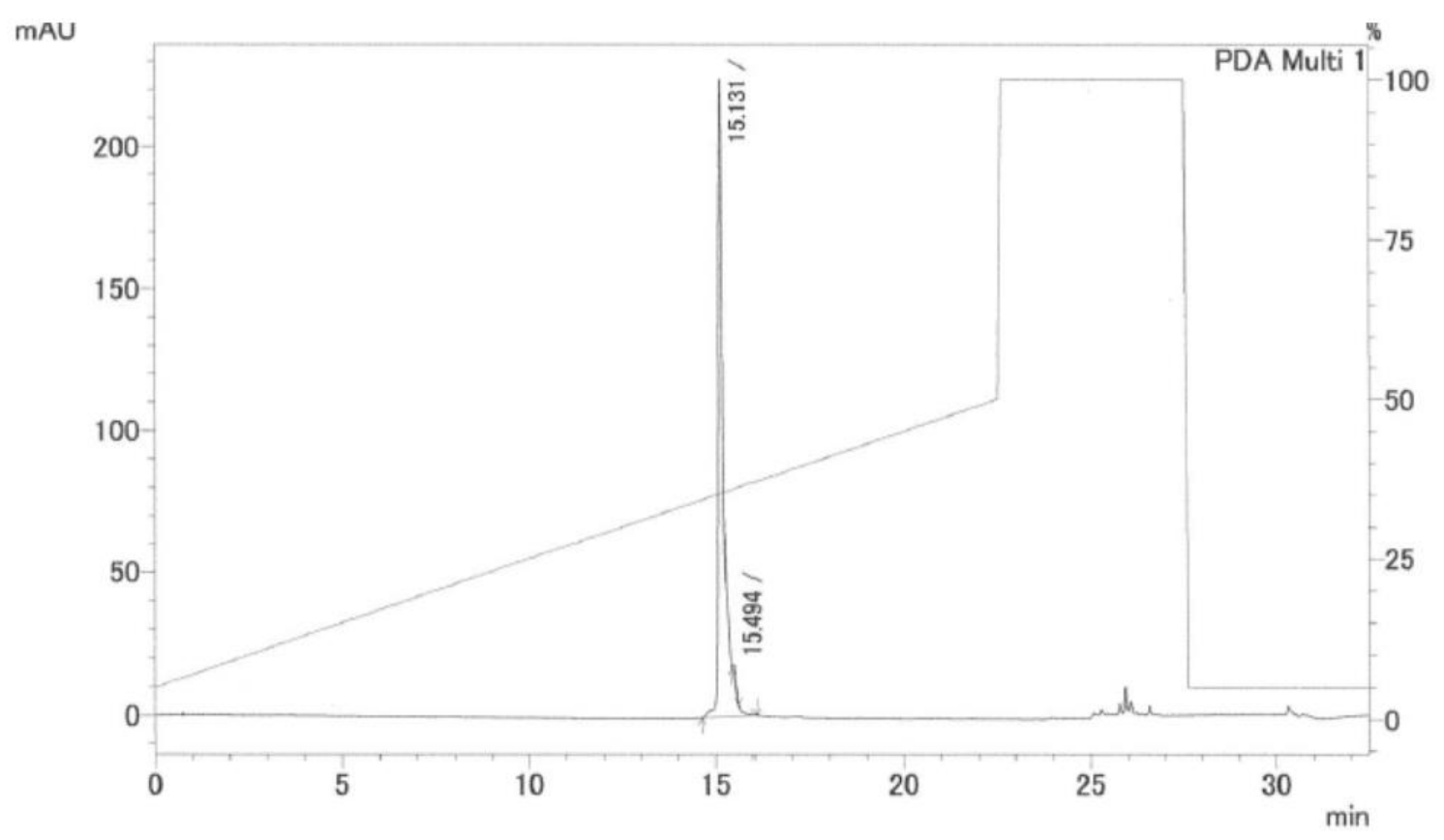

AMO-20

HPLC (gradient condition: B\% 5-50\% in $22.5 \mathrm{~min}$, then $100 \%$ in $5 \mathrm{~min}$ ) 


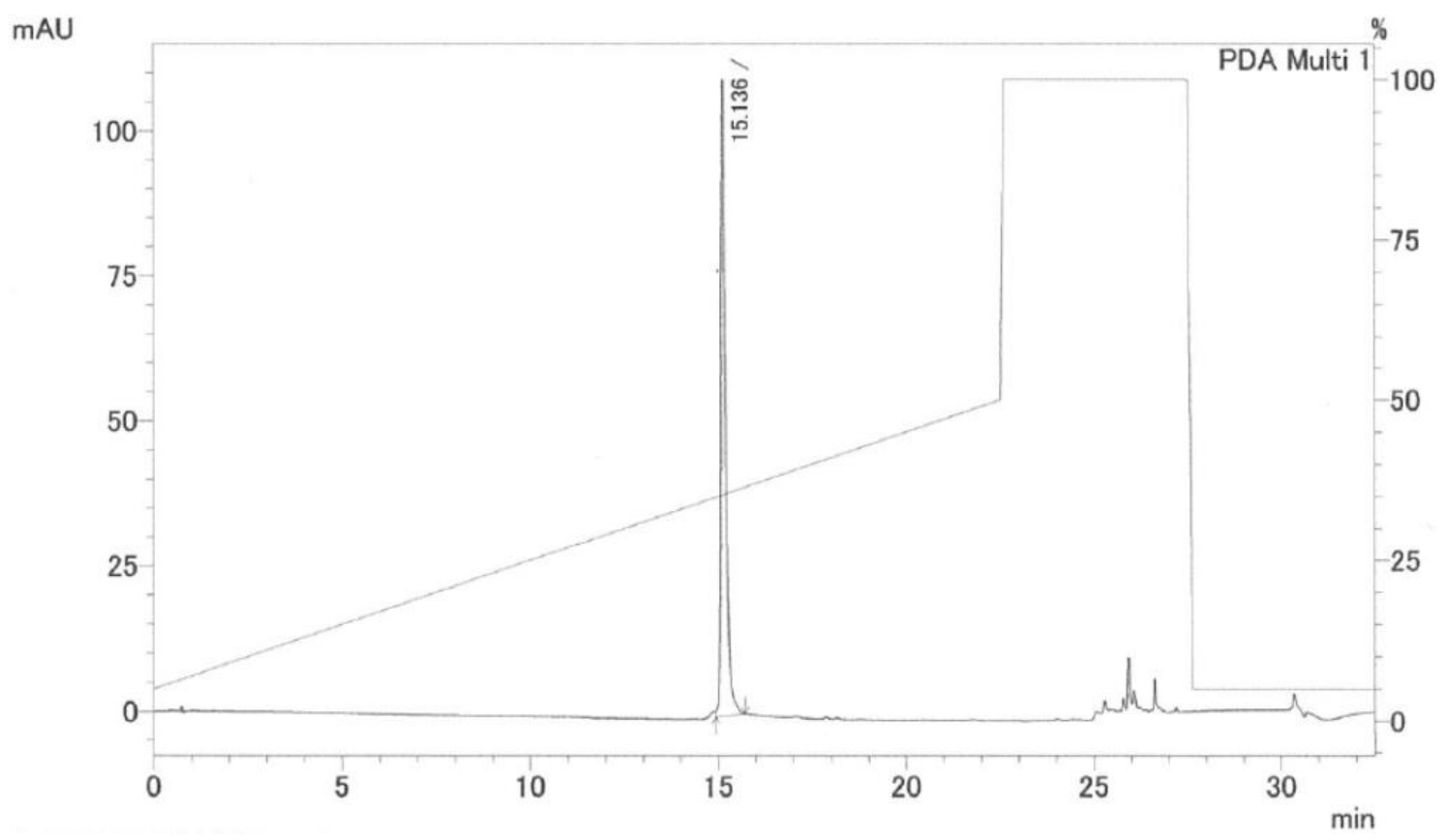

AMO-21

HPLC (gradient condition: B\% 5-50\% in $22.5 \mathrm{~min}$, then $100 \%$ in $5 \mathrm{~min}$ )

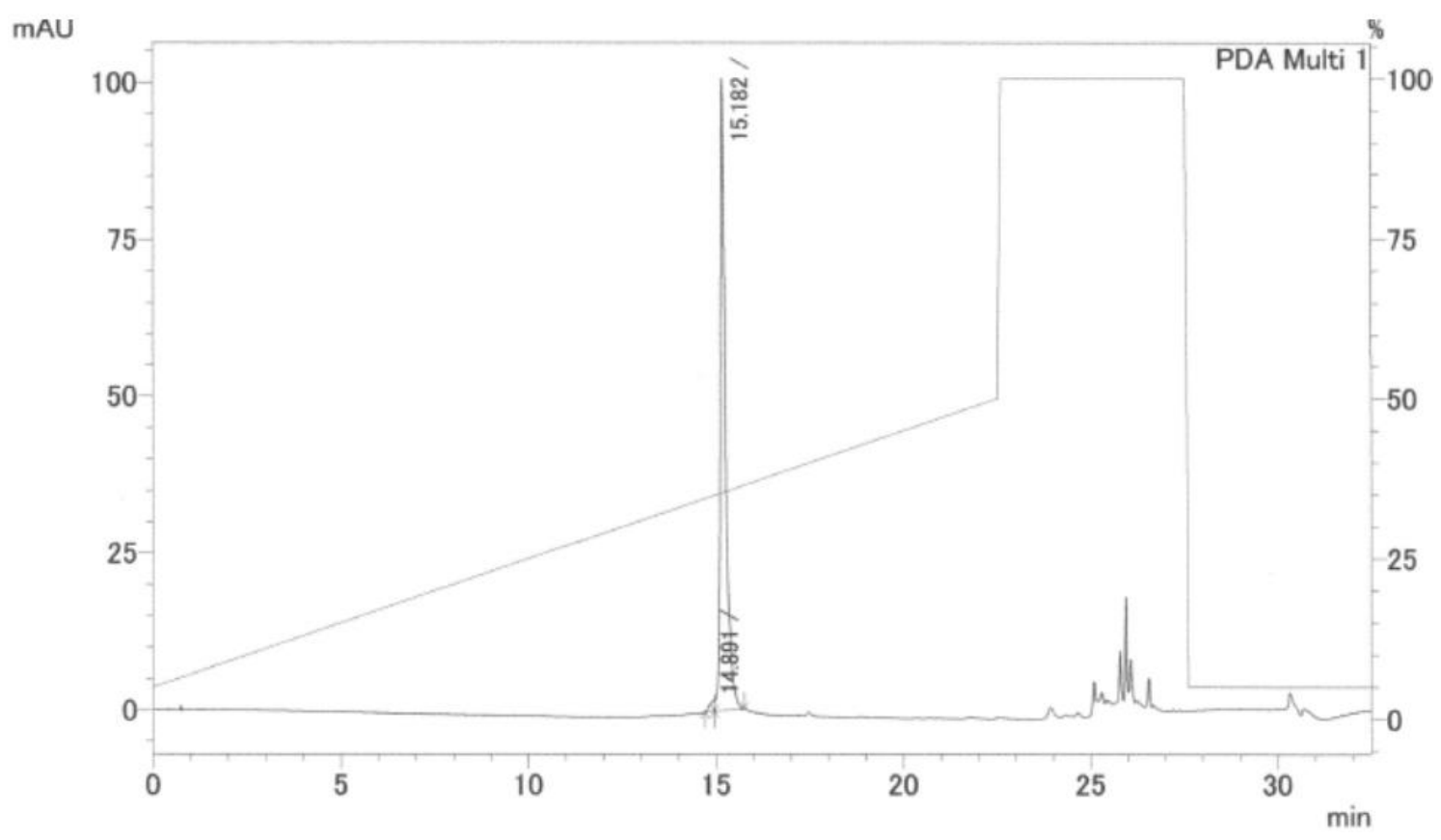

AMO-22

HPLC (gradient condition: B\% 5-50\% in $20 \mathrm{~min}$, then $100 \%$ in $3 \mathrm{~min}$ ) 


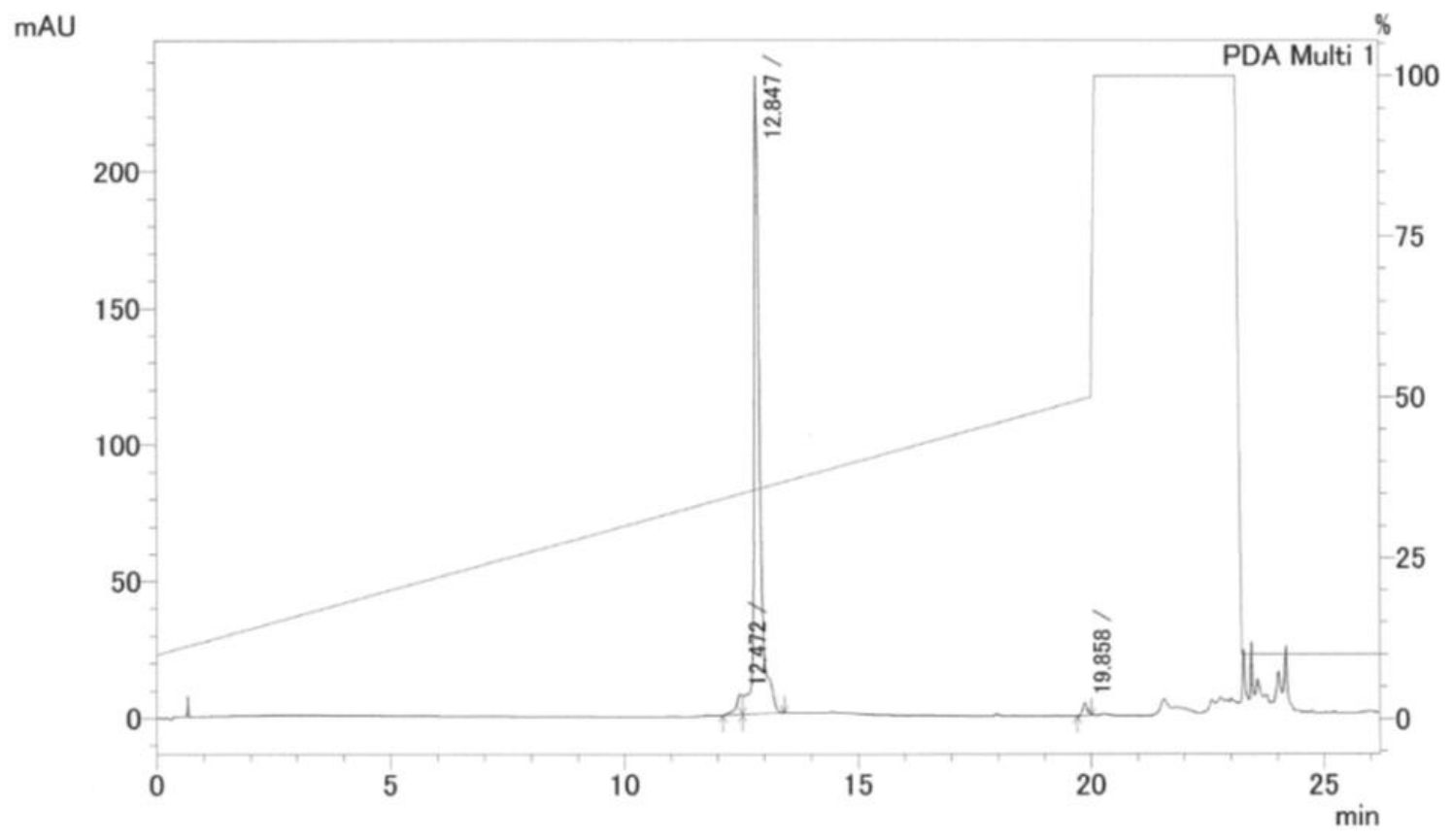

AMO-23

HPLC (gradient condition: B\% 5-50\% in $22.5 \mathrm{~min}$, then $100 \%$ in $5 \mathrm{~min}$ )

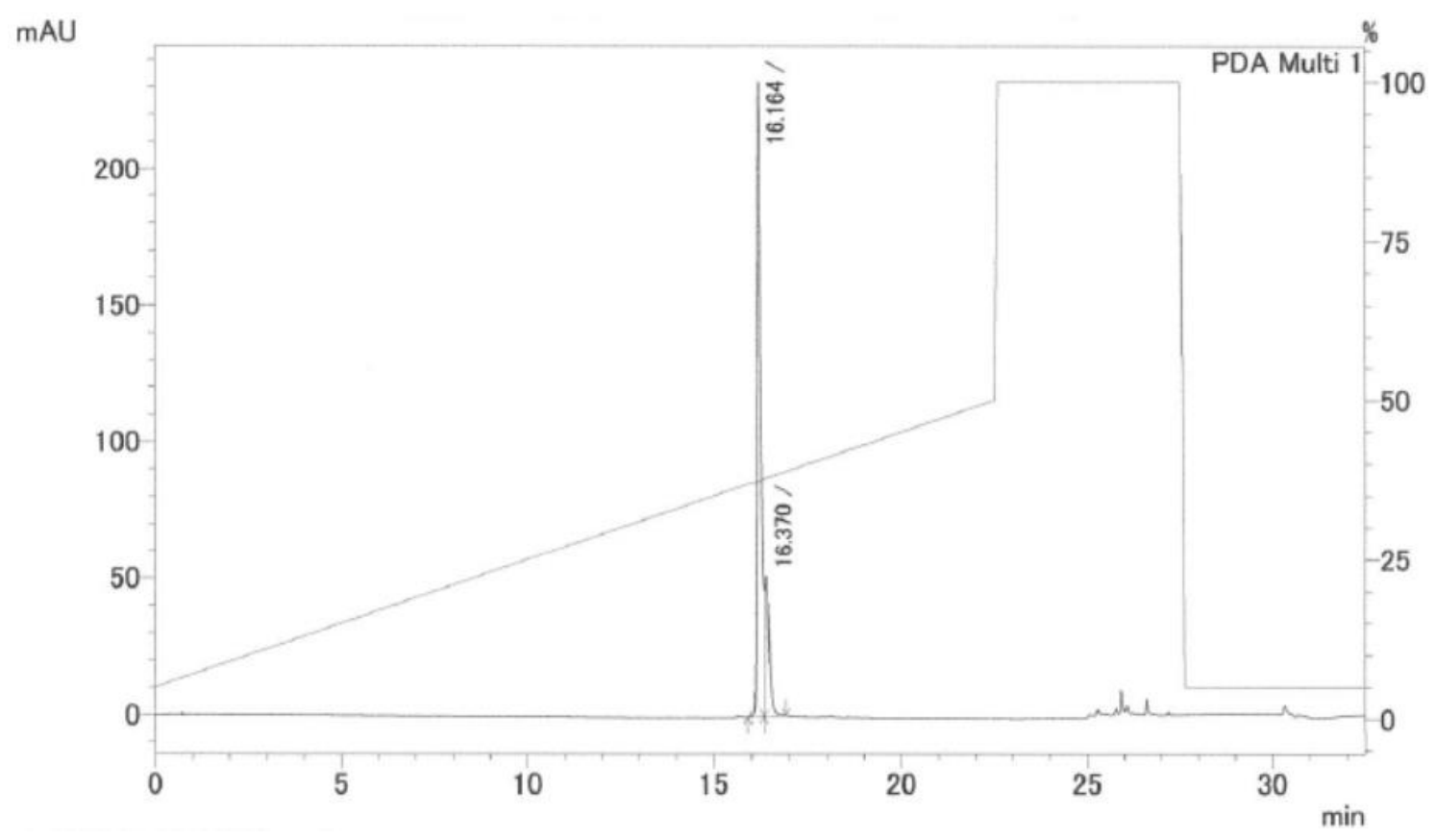

AMO-24

HPLC (gradient condition: B\% 5-50\% in $22.5 \mathrm{~min}$, then $100 \%$ in $5 \mathrm{~min}$ ) 


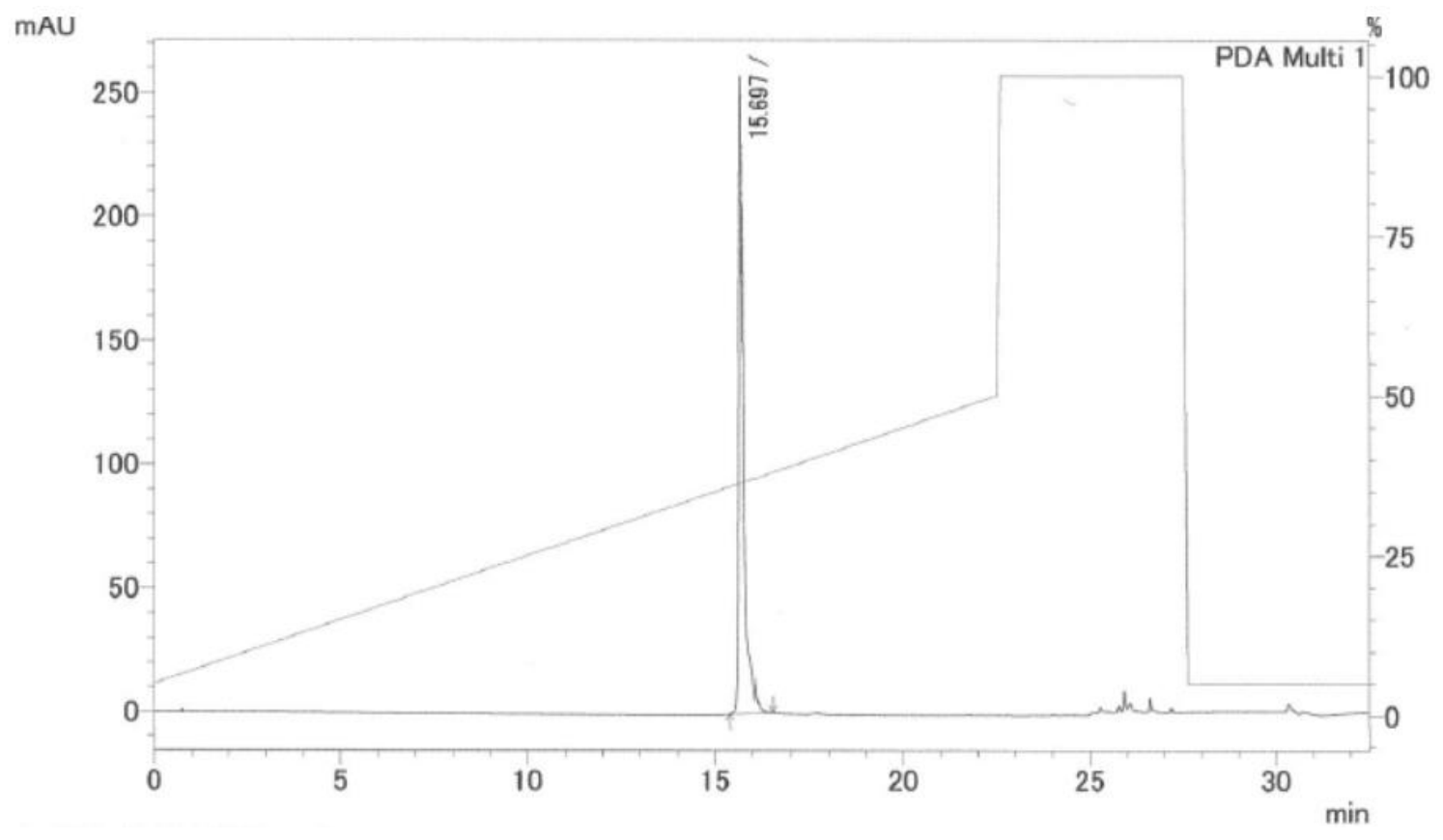

AMO-25

HPLC (gradient condition: B\% 5-50\% in $20 \mathrm{~min}$, then $100 \%$ in $3 \mathrm{~min}$ )

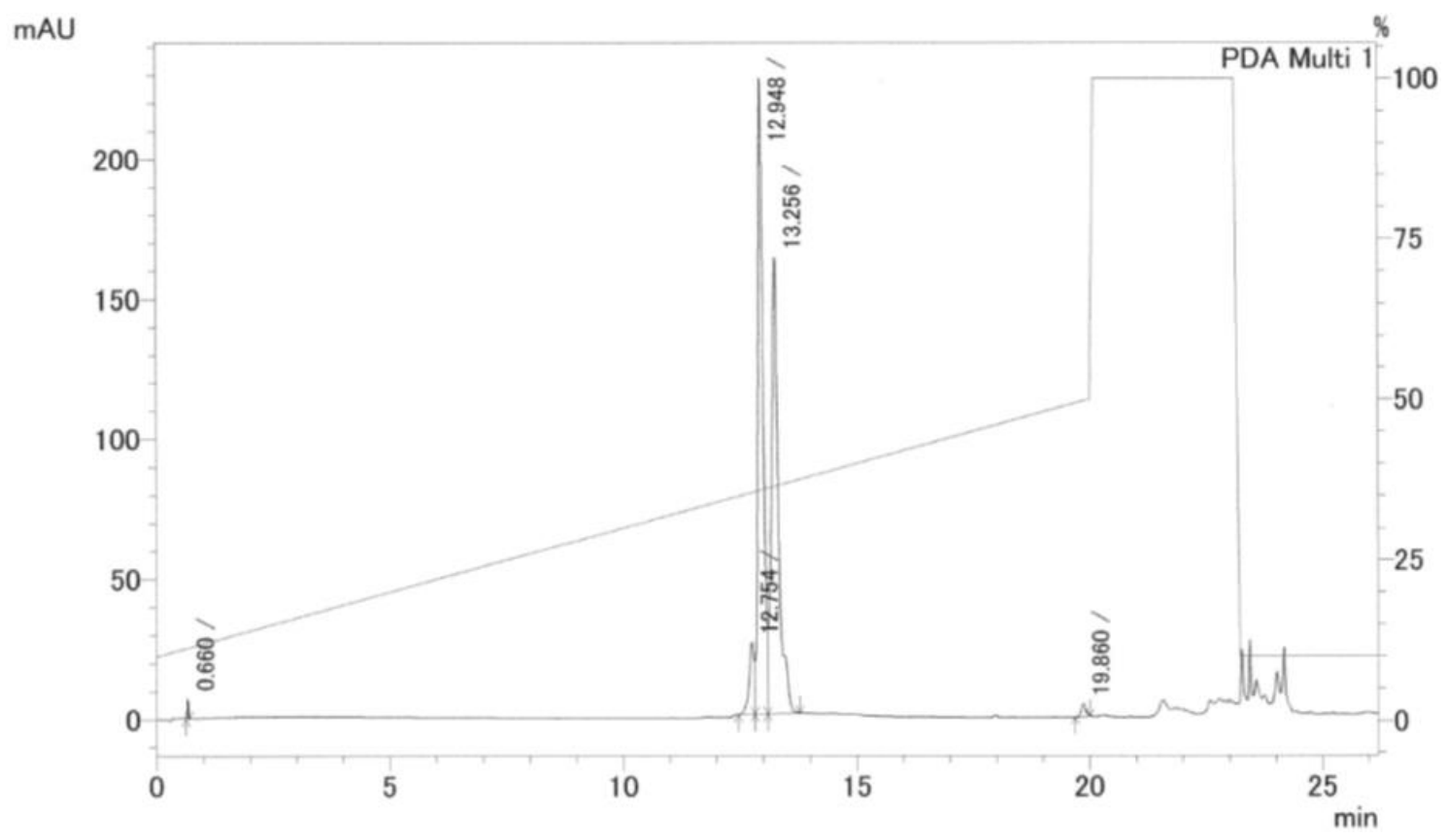

AMO-26

HPLC (gradient condition: B\% 5-50\% in 20 min, then $100 \%$ in $3 \mathrm{~min}$ ) 


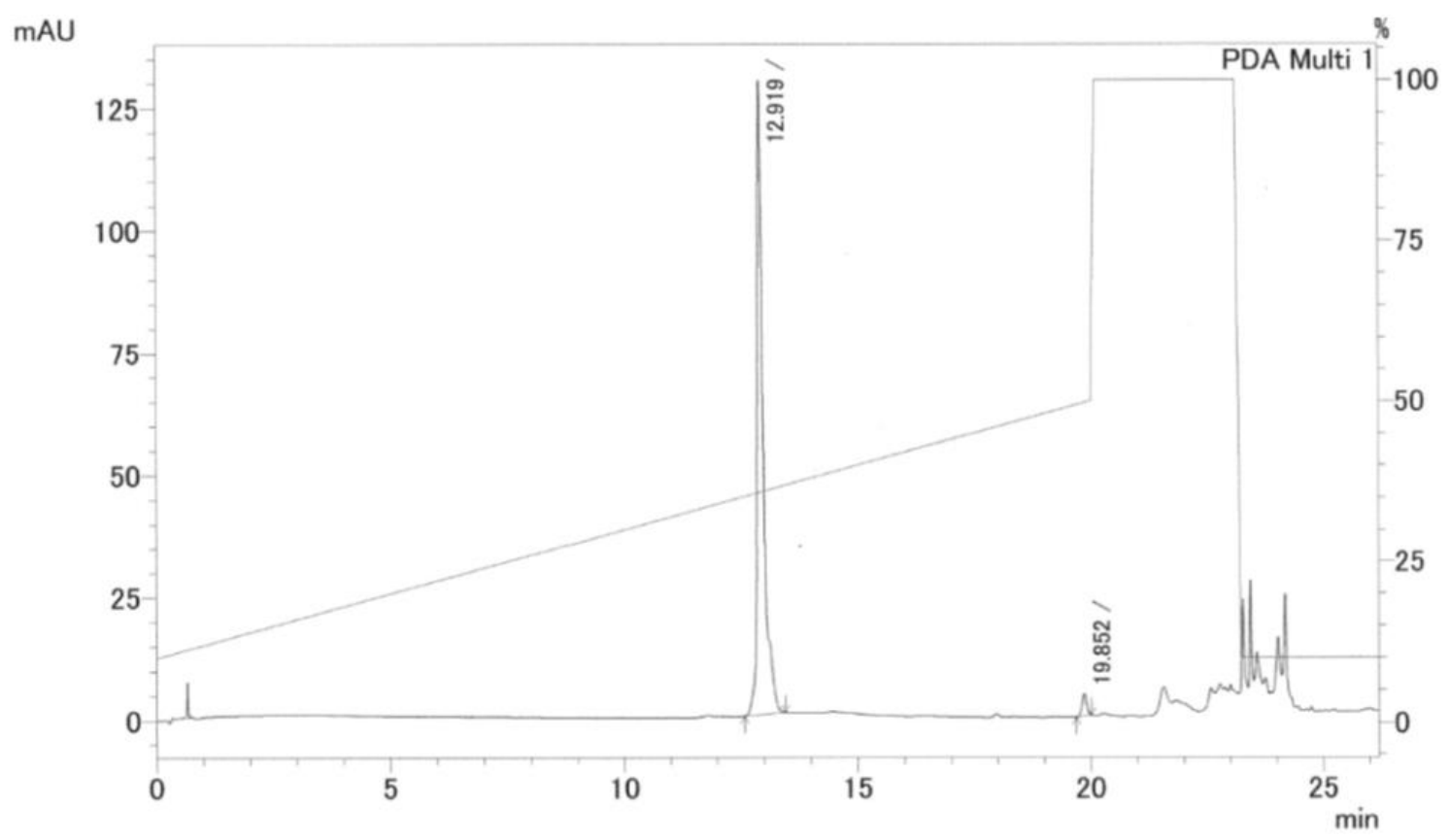

AMO-27

HPLC (gradient condition: B\% 5-50\% in $20 \mathrm{~min}$, then $100 \%$ in $3 \mathrm{~min}$ )

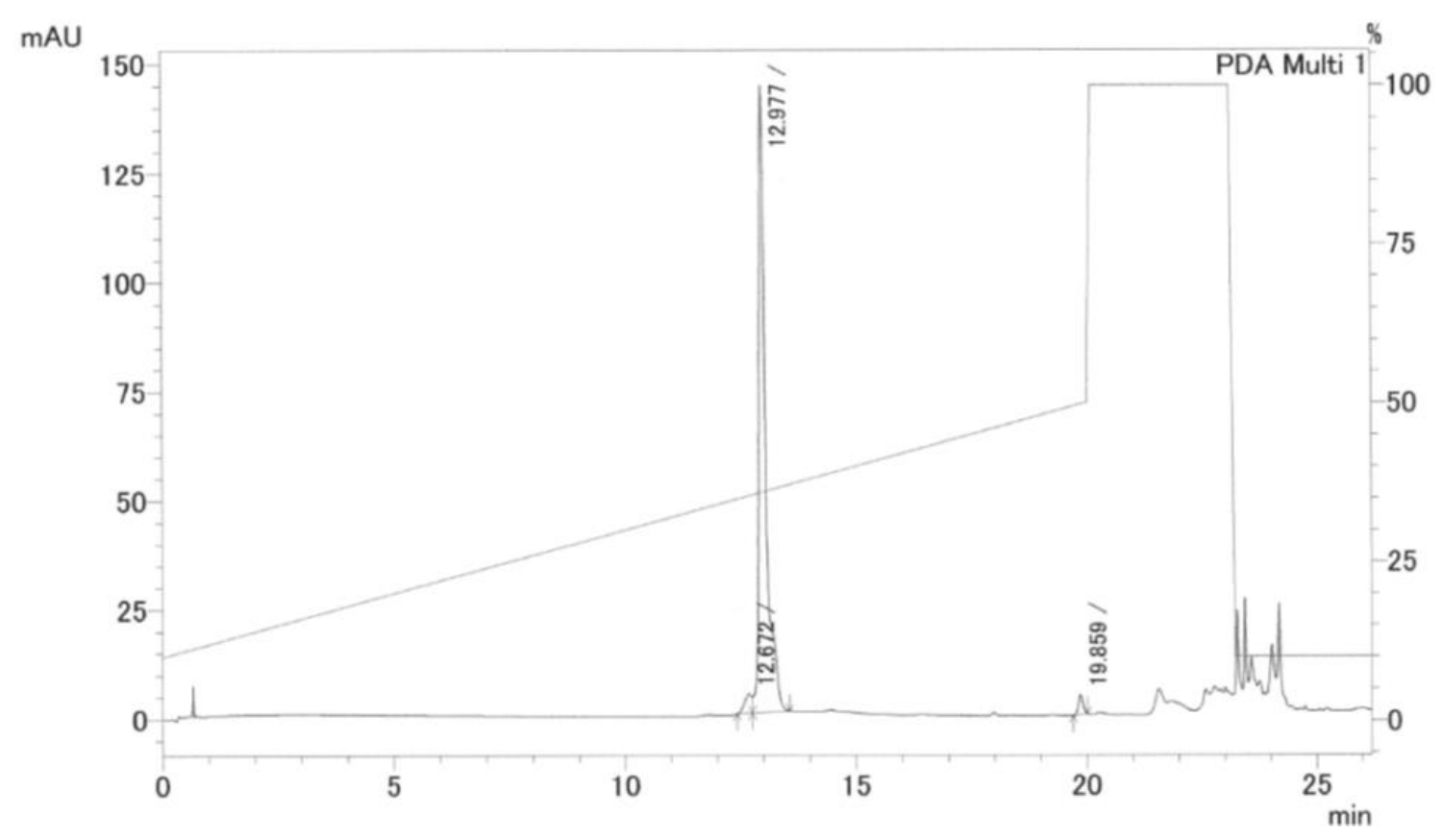

AMO-28

HPLC (gradient condition: B\% 5-50 \% in $20 \mathrm{~min}$, then $100 \%$ in $3 \mathrm{~min}$ ) 


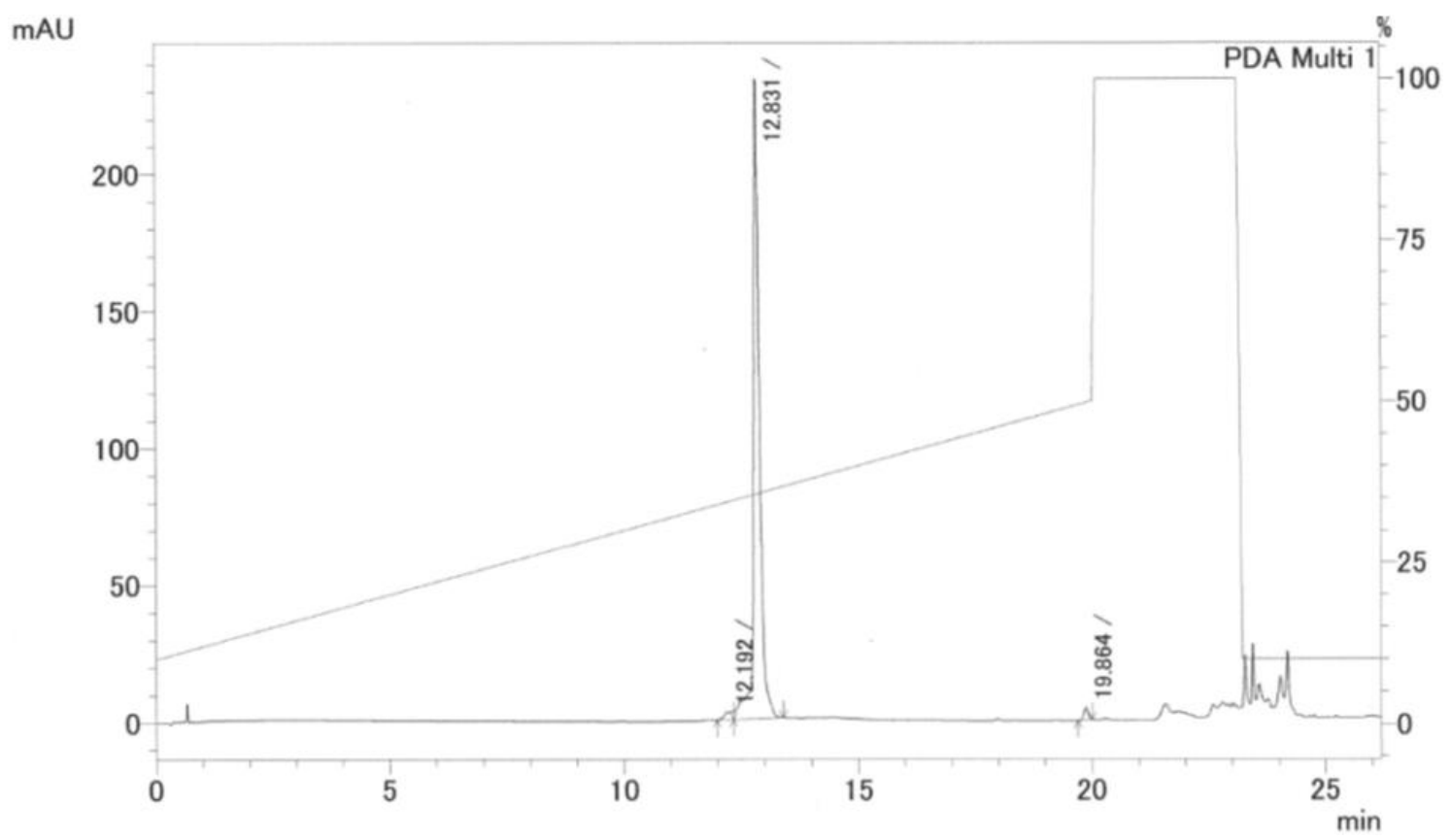

AMO-29

HPLC (gradient condition: B\% 5-50\% in $20 \mathrm{~min}$, then $100 \%$ in $3 \mathrm{~min}$ )

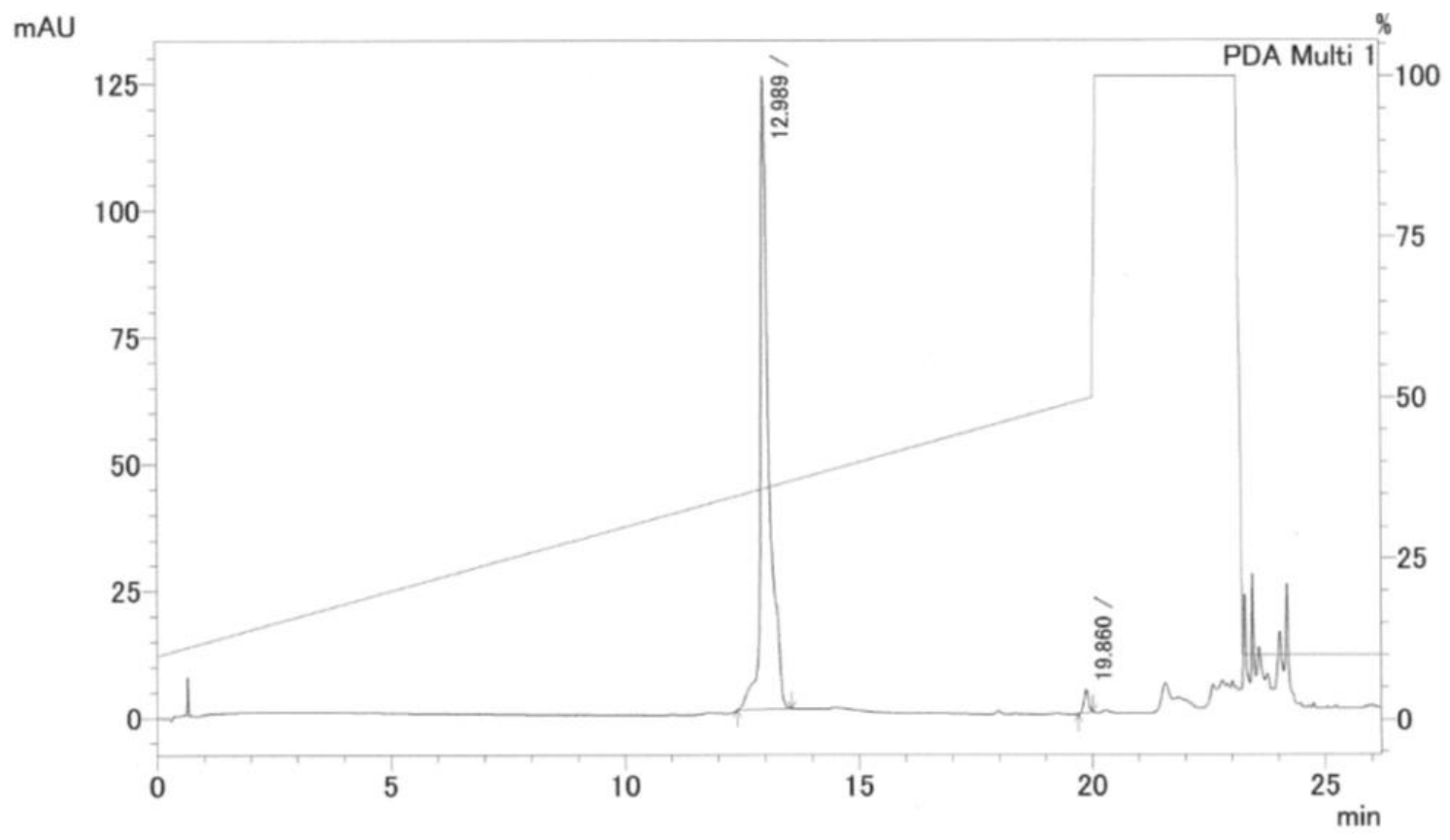

AMO-30

HPLC (gradient condition: B\% 5-50\% in $20 \mathrm{~min}$, then $100 \%$ in $3 \mathrm{~min}$ ) 


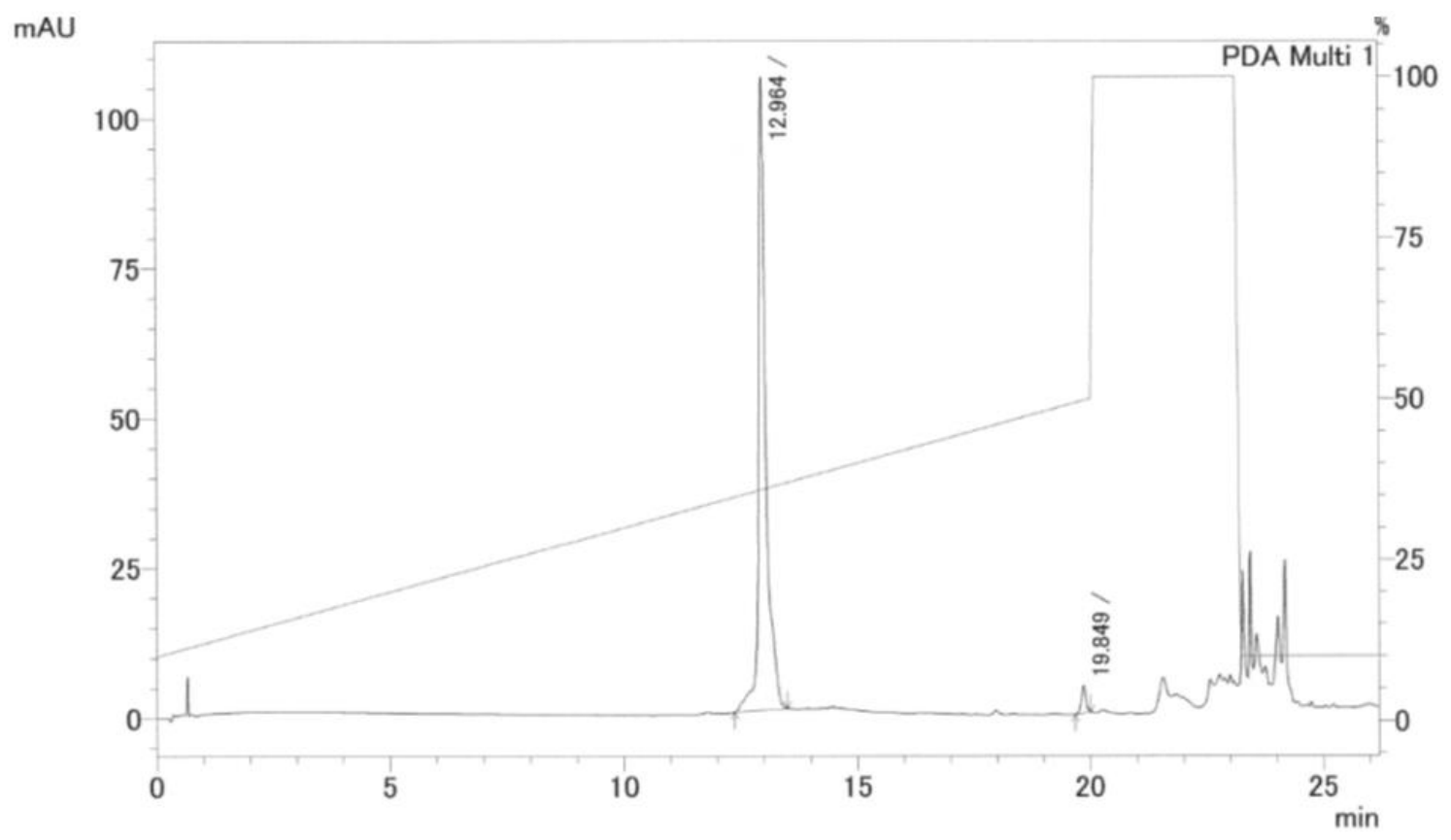

AMO-31

HPLC (gradient condition: B\% 5-50\% in $20 \mathrm{~min}$, then $100 \%$ in $3 \mathrm{~min}$ )

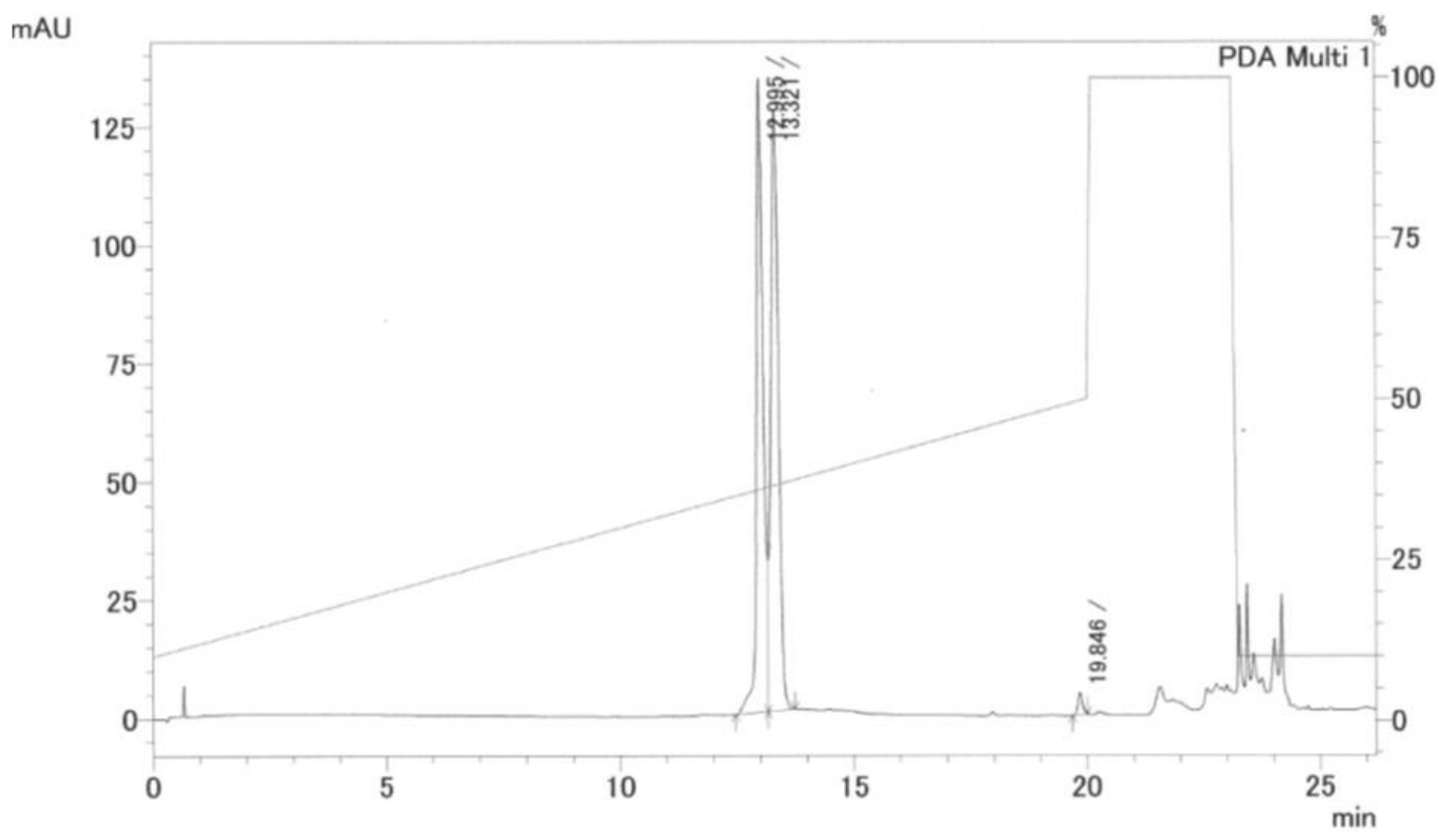

AMO-32

HPLC (gradient condition: B\% 5-50 \% in $20 \mathrm{~min}$, then $100 \%$ in $3 \mathrm{~min}$ ) 


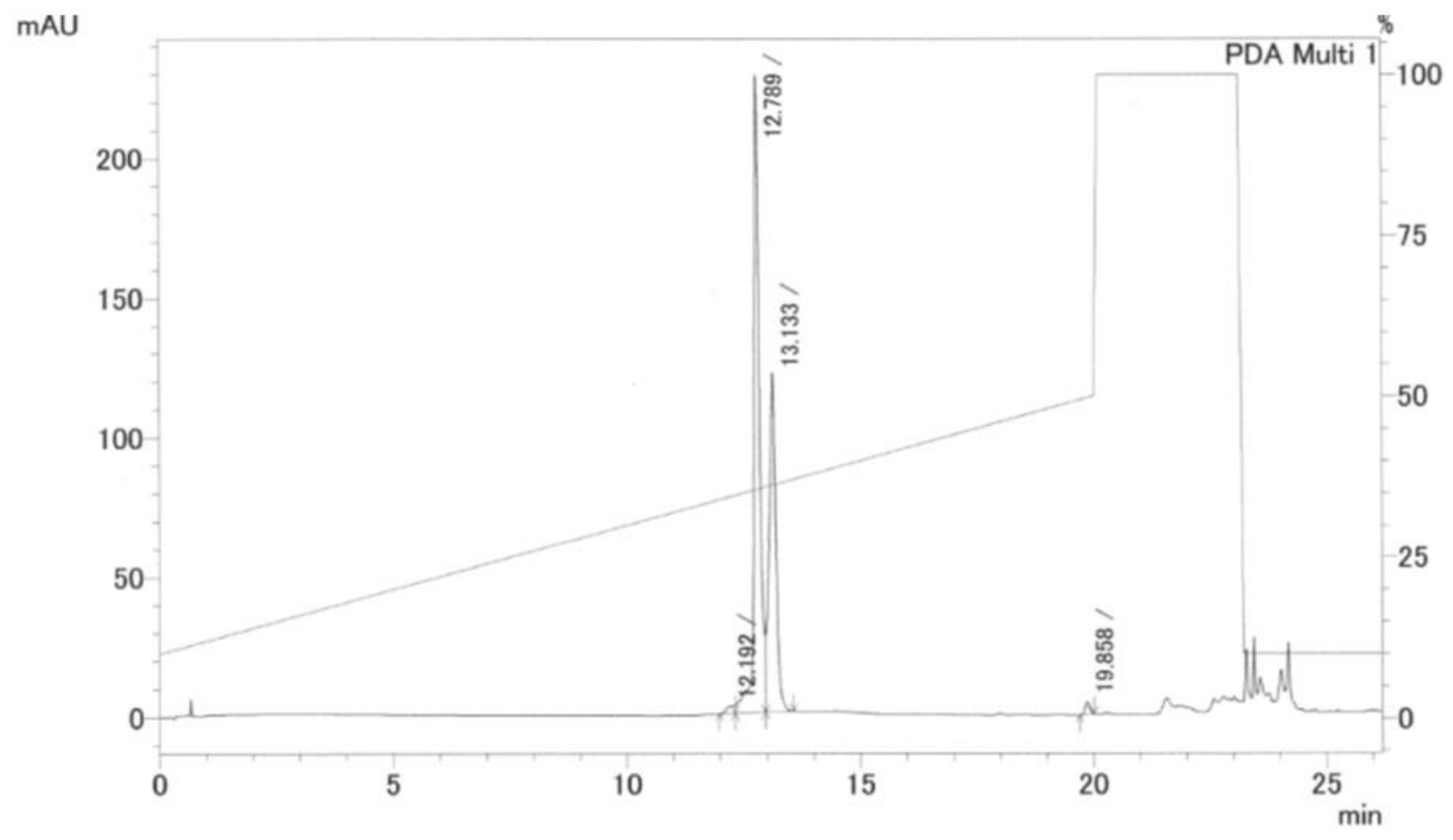

AMO-33

HPLC (gradient condition: B\% 5-50 \% in $20 \mathrm{~min}$, then 100\% in $3 \mathrm{~min}$ )

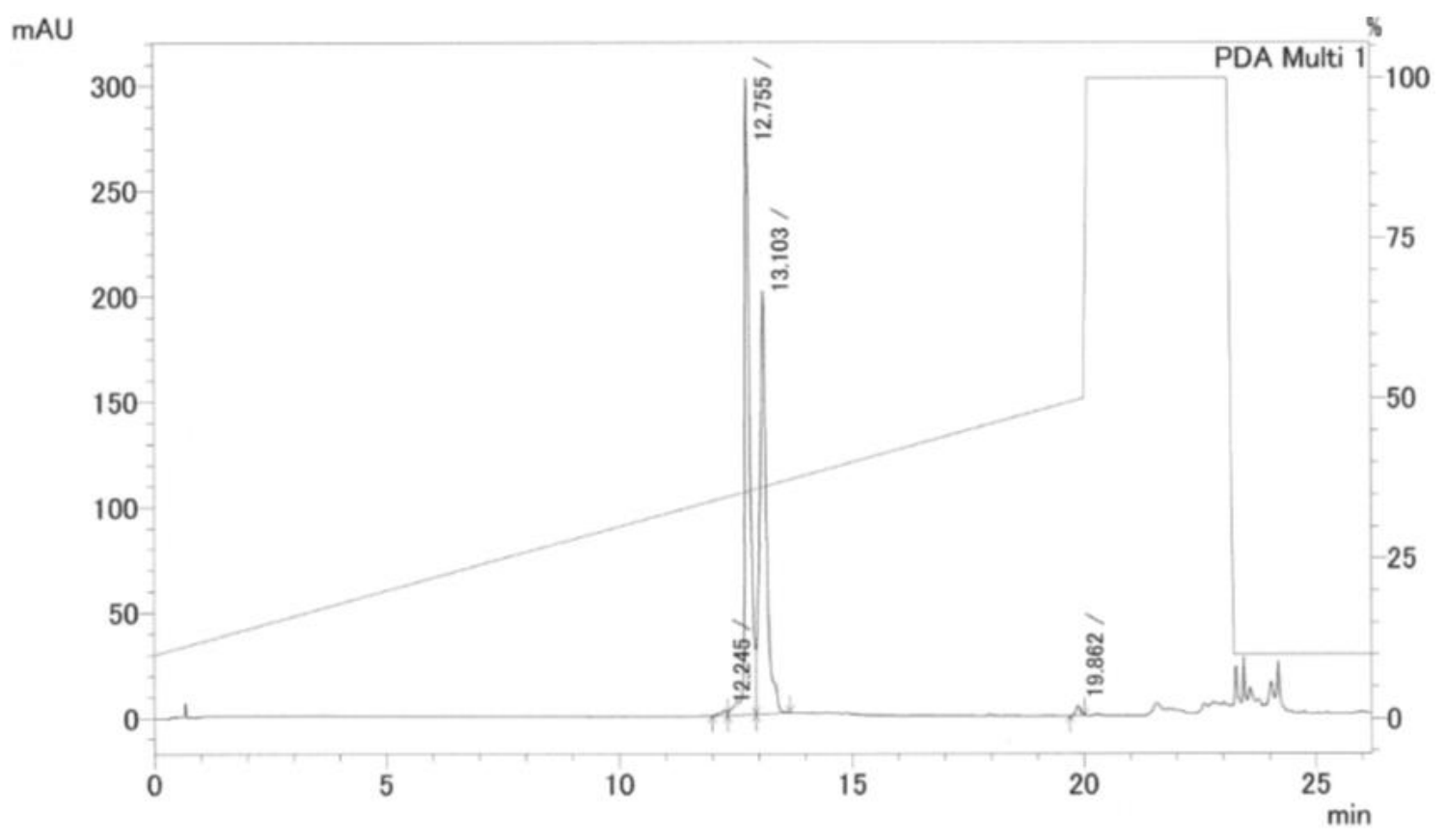




\section{Representative UV melting curves}
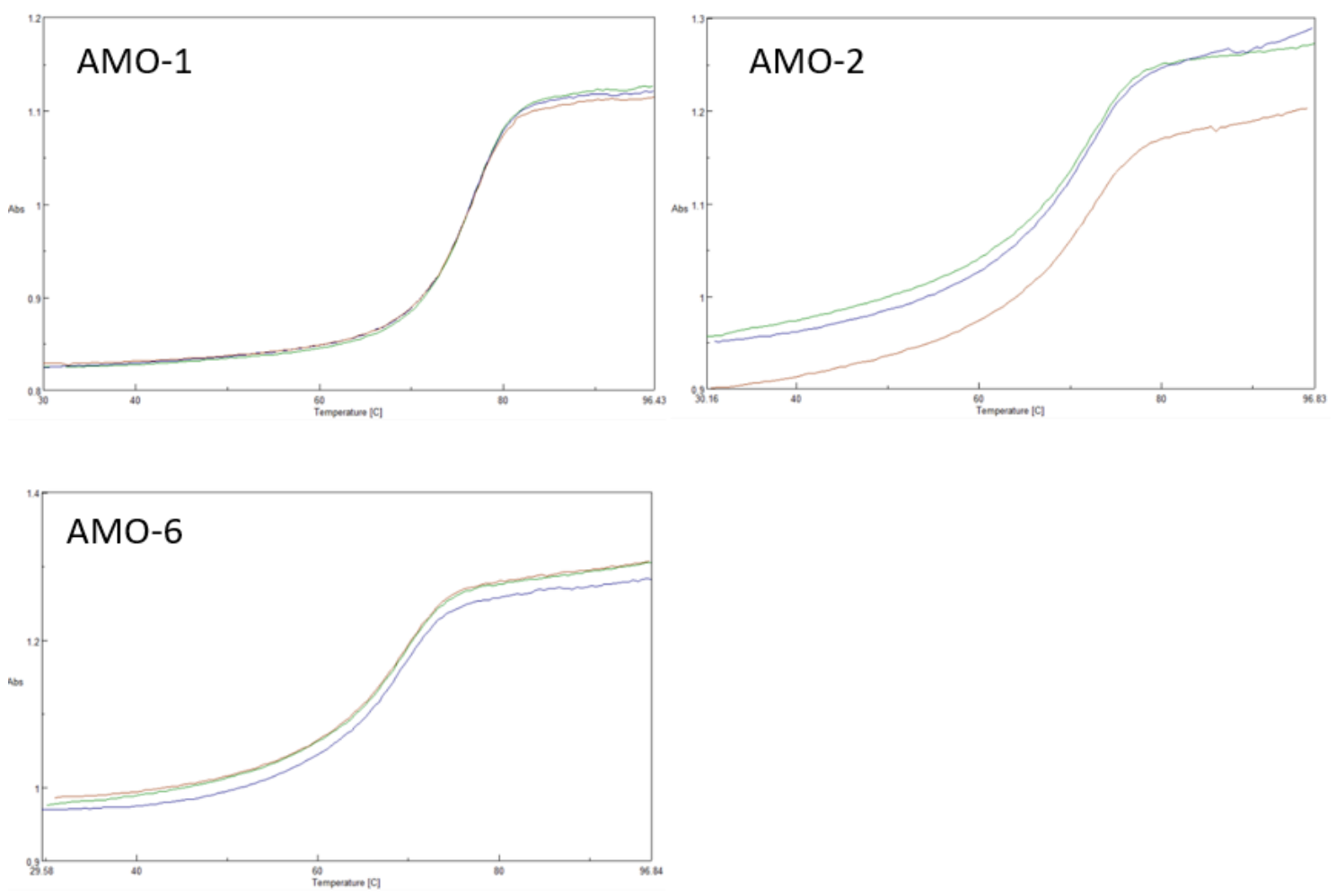

Figure S-1. Thermal stability of the duplexes formed between GuNA-modified oligonucleotides and fully-matched ssRNA. Experiments were performed at least three times independently per sequence.

\begin{tabular}{|c|c|c|c|c|}
\hline Seq No. & Sequence (5' to 3') & Modification & $\begin{array}{c}\mathrm{Ct} \\
\text { (umol/L) }\end{array}$ & $\begin{array}{r}T_{\mathrm{m}}\left[{ }^{\circ} \mathrm{C}\right] \text { toward ssRNA } \\
5^{\prime} \text {-r(UAGCUUAUCAGACUGAUGUUGA)-3' }\end{array}$ \\
\hline AMO-1 & AaCatCagTctGatAagCT & LNA & 2 & $76.43 \pm 0.0$ \\
\hline AMO-2 & AaCatCagTctGatAagCT & GuNA & 2 & $71.58 \pm 0.2$ \\
\hline AMO-6 & AAcaTcaGtcTgaTaaGcT & GuNA & 2 & $68.79 \pm 0.1$ \\
\hline
\end{tabular}

Table S-2. Tm values were calculated as the average of three independent experiments for each sequence. 


\section{Analysis of the crystal structure of ternary complex}

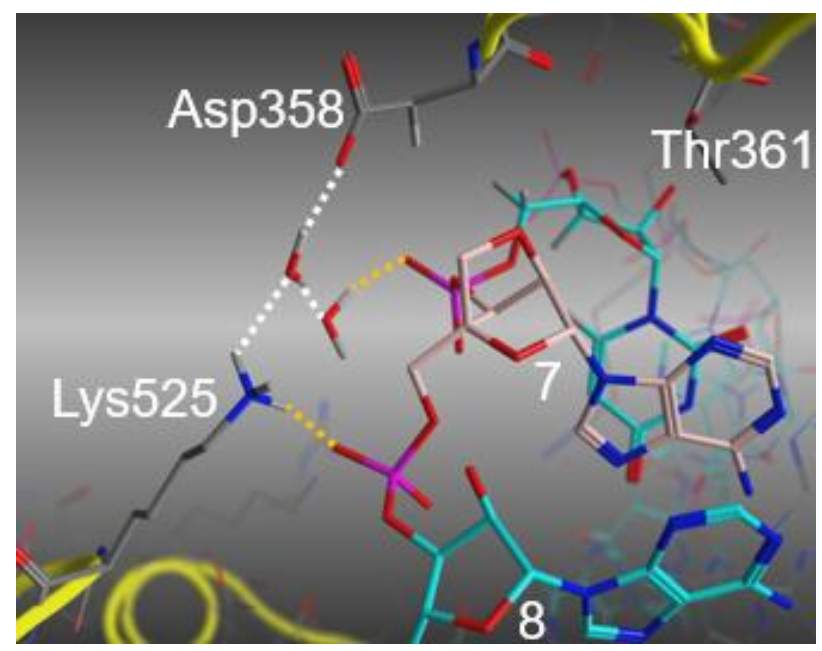

Figure S-2. Energy-minimized structure of the ternary complex with LNA substituted at position 7 of the target RNA. 